\title{
LONG-TIME DYNAMICS OF THE NONHOMOGENEOUS INCOMPRESSIBLE FLOW OF NEMATIC LIQUID CRYSTALS*
}

\author{
XIANPENG $\mathrm{HU}^{\dagger}$ AND HAO $\mathrm{WU}^{\ddagger}$
}

\begin{abstract}
We study the long-time behavior of global strong solutions to a hydrodynamic system for nonhomogeneous incompressible nematic liquid crystal flows driven by two types of external forces in a smooth bounded domain of dimension two. For arbitrary large regular initial data with the initial density being away from vacuum, we prove the decay of the velocity field for both cases. Furthermore, for the case with asymptotically autonomous external force, we can prove the convergence of the density function and the director vector as time goes to infinity. Estimates on the convergence rate are also provided.
\end{abstract}

Key words. Nonhomogeneous nematic liquid crystal flow, long-time behavior, uniqueness of asymptotic limit, convergence rate.

AMS subject classifications. 35B40, 35B41, 35Q35, 76D05.

\section{Introduction}

Liquid crystals are substances that exhibit a phase of matter that has properties between those of a conventional liquid and those of a solid crystal [9]. The hydrodynamic theory of liquid crystals due to Ericken and Leslie was developed around the 1960 's $[10,19,20]$. Since then, the mathematical theory is still progressing and the study of the full Ericksen-Leslie model presents relevant mathematical difficulties. We consider the following hydrodynamical model for the flow of nematic liquid crystals (cf. [22]):

$$
\begin{aligned}
\rho_{t}+v \cdot \nabla \rho & =0, \\
\rho\left(v_{t}+v \cdot \nabla v\right)-\nu \Delta v+\nabla P & =-\lambda \nabla \cdot(\nabla d \odot \nabla d)+\rho \mathbf{g}, \\
\nabla \cdot v & =0, \\
d_{t}+v \cdot \nabla d & =\gamma(\Delta d-f(d)),
\end{aligned}
$$

in $\Omega \times \mathbb{R}^{+}$, where $\Omega \subset \mathbb{R}^{n}(n=2,3)$ is assumed to be a bounded domain with smooth boundary $\Gamma$. System (1.1)-(1.4) is subject to the Dirichlet boundary conditions

$$
v(x, t)=0, \quad d(x, t)=d_{0}(x), \quad \text { for }(x, t) \in \Gamma \times \mathbb{R}^{+},
$$

and the initial conditions

$$
\left.\rho\right|_{t=0}=\rho_{0}(x),\left.\quad v\right|_{t=0}=v_{0}(x) \quad \text { with } \nabla \cdot v_{0}=0,\left.\quad d\right|_{t=0}=d_{0}(x), \quad \text { for } x \in \Omega .
$$

In the above system, $\rho$ is the density of the material, $v$ is the velocity field of the flow, and $d$ represents the averaged macroscopic/continuum molecular orientation in $\mathbb{R}^{n}$. $P(x, t)$ is a scalar function representing the pressure (including both the hydrostatic and the induced elastic part from the orientation field). g stands for the external

*Received: May 17, 2012; accepted (in revised form): September 18, 2012. Communicated by Chun Liu.

${ }^{\dagger}$ Courant Institute of Mathematical Sciences, New York University, New York, NY 10012, USA (xianpeng@cims.nyu.edu).

$\ddagger$ Corresponding author. School of Mathematical Sciences and Shanghai Key Laboratory for Contemporary Applied Mathematics, Fudan University, Shanghai 200433, P.R. China (haowufd@yahoo.com). 
body force. The positive constants $\nu, \lambda$, and $\gamma$ stand for viscosity, the competition between kinetic energy and potential energy, and macroscopic elastic relaxation time (Debroah number) for the molecular orientation field. We assume that $f(d)=\nabla F(d)$ for some smooth bounded function $F: \mathbb{R}^{n} \rightarrow \mathbb{R}$. $\quad \nabla d \odot \nabla d$ denotes the $n \times n$ matrix whose $(i, j)$-th entry is given by $\nabla_{i} d \cdot \nabla_{j} d$, for $1 \leq i, j \leq n$.

System (1.1)-(1.4) is a nonhomogeneous version of the following simplified system introduced in $[22,25]$ that models the incompressible flow of nematic liquid crystals with varying director lengths:

$$
\begin{aligned}
v_{t}+v \cdot \nabla v-\nu \Delta v+\nabla P & =-\lambda \nabla \cdot(\nabla d \odot \nabla d), \\
\nabla \cdot v & =0, \\
d_{t}+v \cdot \nabla d & =\gamma(\Delta d-f(d)) .
\end{aligned}
$$

System (1.7)-(1.9) keeps the important mathematical structure as well as some of the essential features of the original Ericksen-Leslie system. The Ginzburg-Landau approximation

$$
f(d)=\frac{1}{\eta^{2}}\left(|d|^{2}-1\right) d, \text { with its antiderivative } F(d)=\frac{1}{4 \eta^{2}}\left(|d|^{2}-1\right)^{2},
$$

was introduced in order to relax the nonlinear constraint $|d|=1$. The system (1.7)(1.9) has been studied in a series of works not only theoretically $[25,26,29,42]$ but also numerically $[28,30]$ (see also [15] for the case $f(d)=0$ ). In [25], the authors proved the existence of global weak solutions to system (1.7)-(1.9) with Dirichlet boundary conditions by a semi-Galerkin method. Global existence and uniqueness of classical solutions to the same system was proved for $n=2$ or $n=3$ under an assumption of large viscosity. Long-time behavior of global solutions to system (1.7)-(1.9) was studied in $[25,35,42]$. In particular, convergence of global classical solutions to single steady states as time goes to infinity was obtained in $[35,42]$. We refer to $[3,6,12,29]$ for results on the homogeneous system (1.7)-(1.9) subject to other types of boundary conditions.

As far as the density-dependent system (1.1)-(1.4) is concerned, the authors in $[14,33,43]$ proved existence of global weak solutions of the problem (1.1)-(1.6) without assuming the positive lower bound for the initial density. The basic idea of their proof is to introduce a viscous term $\epsilon \Delta \rho$ in the transport equation (1.1), and then pass to the limit as $\epsilon \rightarrow 0$. In the recent work [7], instead of introducing the viscosity term in (1.1), the authors provided an alternative proof for the existence of global weak solutions to system (1.1)-(1.6) under the stronger Assumption (1.11) (i.e., the initial density is positive and bounded). Regularity properties of weak solutions to system (1.1)(1.6) were proved by using Ladyzhenskaya type energy estimates for the approximate solutions constructed within a proper Galerkin scheme, provided that the initial data are regular and satisfy assumptions (1.11)-(1.12). For a compressible version of the liquid crystal system (1.1)-(1.6), existence and large-time behavior of a global weak solution were established in $[5,32,40]$ while existence of local strong solutions was obtained in [34] (see also [31] for a blow-up criterion). Finally, we refer to the recent works $[4,17,21,24,27,39,41,44]$ and the references cited therein for mathematical results on the liquid crystal system under the constraint $|d|=1$.

We note that the external force $\rho \mathbf{g}$ is supposed to be vanishing in the above mentioned work. In this paper, we focus on the two-dimensional case $n=2$ and extend the results on long-time behavior of global classical solutions in $[25,42]$ to the 
nonhomogeneous system (1.1)-(1.4) with non-vanishing external forces. Two types of external forces will be treated in the following text:

(F1) $\mathbf{g}$ is a time-independent potential field, namely,

$$
\mathbf{g}=\nabla \phi, \quad \text { for some scalar function } \phi(x) \in H^{2}(\Omega) .
$$

(F2) $\mathrm{g}$ depends on time and satisfies the following integrability conditions:

$$
\mathbf{g} \in L^{2}\left(0,+\infty ; \mathbf{H}^{1}(\Omega)\right), \quad \mathbf{g}_{t} \in L^{2}\left(0,+\infty ; \mathbf{L}^{2}(\Omega)\right) .
$$

The main results of this paper are as follows.

TheOREM 1.1. Suppose that $r \in(2,+\infty)$, and that the external force $\mathbf{g}$ satisfies either (F1) or (F2). For any initial data $\rho_{0} \in W^{1, r}(\Omega), v_{0} \in \mathbf{H}^{2}(\Omega) \cap V$, and $d_{0} \in \mathbf{H}^{3}(\Omega)$ that satisfy

$$
\begin{aligned}
& 0<\underline{\rho} \leq \rho_{0}(x) \leq \bar{\rho}, \quad \forall x \in \Omega, \\
& \left|d_{0}(x)\right| \leq 1, \quad \forall x \in \Omega,
\end{aligned}
$$

where $\underline{\rho}$ and $\bar{\rho}$ are positive constants, problem (1.1)-(1.6) admits a unique global strong solution $(\rho, v, d)$ such that for any $T>0$,

$$
\begin{aligned}
& \rho \in C\left([0, T], W^{1, r}(\Omega)\right), \\
& v \in C\left([0, T] ; \mathbf{H}^{2}(\Omega) \cap V\right) \cap L^{2}\left(0, T ; \mathbf{H}^{3}\right), \quad v_{t} \in L^{2}(0, T ; V), \\
& d \in C\left([0, T] ; \mathbf{H}^{3}(\Omega)\right) \cap L^{2}\left(0, T ; \mathbf{H}^{4}\right), \quad d_{t} \in C\left([0, T] ; \mathbf{H}_{0}^{1}(\Omega)\right) \cap L^{2}\left(0, T ; \mathbf{H}^{2}\right), \\
& 0<\underline{\rho} \leq \rho(x, t) \leq \bar{\rho}, \quad|d(x, t)| \leq 1, \quad \forall(x, t) \in \Omega \times[0, T] .
\end{aligned}
$$

THEOREM 1.2. Suppose that the assumptions of Theorem 1.1 are satisfied with $\mathbf{g}$ fulfilling (F1). The global strong solution to problem (1.1)-(1.6) has the following property:

$$
\lim _{t \rightarrow+\infty}\left(\|v(t)\|_{\mathbf{H}^{1}}+\left\|v_{t}\right\|+\left\|d_{t}\right\|_{\mathbf{H}^{1}}\right)=0 .
$$

For any unbounded sequence $\left\{t_{i}\right\}$, there is a subsequence $\left\{t_{i}^{\prime}\right\} \nearrow+\infty$ such that

$$
\begin{aligned}
& \left\|\rho\left(t_{i}^{\prime}\right)-\rho_{\infty}\right\|_{L^{q}} \rightarrow 0, \quad \text { as } t_{i}^{\prime} \rightarrow+\infty, q \in(1,+\infty), \\
& \left\|d\left(t_{i}^{\prime}\right)-d_{\infty}\right\|_{\mathbf{H}^{3}} \rightarrow 0, \quad \text { as } t_{i}^{\prime} \rightarrow+\infty,
\end{aligned}
$$

where $\rho_{\infty}$ is a certain function that belongs to $L^{q}$ and $d_{\infty}$ is a solution to the following nonlinear elliptic boundary value problem:

$$
\left\{\begin{array}{l}
-\Delta d_{\infty}+f\left(d_{\infty}\right)=0, \quad x \in \Omega \\
d_{\infty}=d_{0}(x), x \in \Gamma
\end{array}\right.
$$

THEOREM 1.3. Suppose that the assumptions of Theorem 1.1 are satisfied with $\mathbf{g}$ fulfilling (F2). If in addition, $\mathbf{g}$ satisfies

$$
\sup _{t \geq 0}(1+t)^{1+\xi} \int_{t}^{+\infty}\|\mathbf{g}(\tau)\|^{2} d \tau<+\infty, \quad \text { for some } \xi>0,
$$

then the global strong solution to problem (1.1)-(1.6) has the following property:

$$
\lim _{t \rightarrow+\infty}\left(\left\|\rho(t)-\rho_{\infty}\right\|_{L^{q}}+\|v(t)\|_{\mathbf{H}^{1}}+\left\|v_{t}\right\|+\left\|d_{t}\right\|_{\mathbf{H}^{1}}+\left\|d(t)-d_{\infty}\right\|_{\mathbf{H}^{3}}\right)=0,
$$


where $\rho_{\infty}$ is certain function in $L^{q}(q \in(1,+\infty))$ and $d_{\infty}$ is a solution of (1.16). Moreover, there exists a positive constant $C$ depending on $v_{0}, d_{0}, \nu, \eta, \Omega, \bar{\rho}, \underline{\rho}, d_{\infty}$, such that

$$
\left\|\rho(t)-\rho_{\infty}\right\|_{H^{-1}}+\|v(t)\|_{\mathbf{H}^{1}}+\left\|d(t)-d_{\infty}\right\|_{\mathbf{H}^{2}} \leq C(1+t)^{-\kappa}, \quad \forall t \geq 0,
$$

where $\kappa=\min \left\{\frac{\theta}{1-2 \theta}, \frac{\xi}{2}\right\}$ with $\theta \in\left(0, \frac{1}{2}\right)$ being a constant depending on $f, d_{\infty}$.

The question on the uniqueness of the asymptotic limit for liquid crystal system (1.7)-(1.9) was raised in [25]. A positive answer was given in [42] such that for any global classical solution $(v, d)$ of the homogeneous system, the velocity field will decay to zero and the director vector will converge to a steady state that is a solution to the stationary problem (1.16) (cf. $[12,35]$ for some generalizations). Decay of the velocity field can be obtained by exploring the dissipative nature of the problem and by proper energy estimates, while convergence of the director vector is a nontrivial problem, because the structure of the equilibria set (namely, the set of solutions to (1.16)) can be quite complicated. The proof in [42] relies on the so-called Łojasiewicz-Simon approach [37], which turns out to be a useful method to study convergence of global solutions to equilibria for nonlinear evolution equations (see e.g., $[2,11,13,16,23]$ and the references cited therein). One advantage of the approach is that one can obtain the convergence result without studying the structure of the set of equilibria, which is usually difficult when the spacial dimension is larger than one.

The nonhomogeneous problem (1.1)-(1.6) under consideration is much more involved than the homogeneous case. The main difficulties come from those nonlinear couplings between the three equations for density, velocity, and director in terms of convection, the extra stress term, as well as the external force. Under both assumptions (F1) and (F2), we are able to derive certain (dissipative) basic energy inequalities for problem (1.1)-(1.6) and, furthermore, some specific higher-order differential inequalities in the sprit of [25], which not only provide uniform-in-time estimates for the global strong solutions but also yield the decay properties of the velocity. Our results on the decay of velocity fields under both types of external forces imply that the dissipations from the viscosity and the relaxation effect in (1.4) are strong enough to compensate for the effects of external forces and the density fluctuation, as well as the interactions between the fluid and the liquid crystal molecules, such that the flow will slow down as time goes to infinity. This extends the result on the densitydependent incompressible Navier-Stokes equations driven by a time-independent external force (cf. (F1)) on bounded domains in 2D (cf. [45]). For the asymptotically autonomous external force (cf. (F2)), we are able to apply the Lojasiewicz-Simon approach to prove convergence of the director vector and thus generalize the previous results in $[25,42]$ for the homogeneous liquid crystal system. In this case, we can also obtain $L^{1}$-integrability of the velocity field, which together with the transport equation (1.1) yields convergence of the density function. Besides, by the EojasiewiczSimon approach we can derive some explicit decay rates of the density, velocity field and director vector. We remark that in the case of time-independent external force, the Eojasiewicz-Simon method seems impossible to apply, and thus we are only able to show certain sequential convergence of the density function and the director vector. Our results still hold in the three-dimensional case provided that bounded global strong solutions of problem (1.1)-(1.6) can be obtained (this could be verified, for instance, if the initial data and the external forces are sufficiently small).

The remaining part of this paper is organized as follows. In Section 2, we introduce the functional settings, some preliminary results, as well as some technical lemmas. In 
Section 3, we derive some dissipative energy inequalities and some specific higher-order differential inequalities for both types of external forces, which enable us to obtain uniform a priori estimates and conclude the existence and uniqueness of global strong solutions to problem (1.1)-(1.6) (cf. Theorem 1.1). Section 4 is devoted to the proof of our main results on the long-time behavior of global strong solutions (cf. Theorem $1.2,1.3)$.

\section{Preliminaries}

As usual, $L^{p}(\Omega)$ and $W^{k, p}(\Omega)$ stand for the Lebesgue and the Sobolev spaces of real valued functions, with the convention that $H^{k}(\Omega)=W^{k, 2}(\Omega)$. The spaces of vector-valued functions are denoted by bold letters, correspondingly. Without any further specification, $\|\cdot\|$ stands for the norm in $L^{2}(\Omega)$ or $\mathbf{L}^{2}(\Omega)$. We shall denote by $C$ the generic constants depending on $\lambda, \gamma, \nu, \eta, \Omega$, and the initial data. Special dependence will be pointed out explicitly in the text if necessary.

For $1<q<+\infty, X^{q}$ denotes the space that is a completion in $\mathbf{L}^{q}$ of the set of solenoidal vector fields with coefficients that belong to $\mathcal{V}=\mathbf{C}_{0}^{\infty}(\Omega) \cap\{v: \nabla \cdot v=0\}$ :

$$
X^{q}=\left\{v \in \mathbf{L}^{q}(\Omega): \nabla \cdot v=0 \text { in } \Omega, v \cdot \mathbf{n}=0 \text { on } \Gamma\right\},
$$

where $\mathbf{n}$ is the unit outer normal to the boundary (cf. [8]). In particular, we denote

$$
H=X^{2}=\text { the closure of } \mathcal{V} \text { in } \mathbf{L}^{2}(\Omega), \quad V=\text { the closure of } \mathcal{V} \text { in } \mathbf{H}_{0}^{1}(\Omega) .
$$

It is well-known that any vector-field with coefficients in $\mathbf{L}^{q}$ has a Helmholtz decomposition (cf. [36]). Denote by $\mathrm{P}_{q}: \mathbf{L}^{q}(\Omega) \rightarrow X^{q}$ the projector from $\mathbf{L}^{q}$ to $X^{q}$, which is a bounded operator. We can define the Stokes operator $A_{q}=\mathrm{P}_{q}(-\Delta)$ with domain $D\left(A_{q}\right)=\mathbf{W}^{2, q} \cap \mathbf{W}_{0}^{1, q} \cap X^{q}$. The following result holds (cf. e.g., [8]).

Lemma 2.1. Let $\Omega$ be a bounded domain of $\mathbb{R}^{2}$ with smooth boundary and $d(\Omega)$ be the diameter of $\Omega$. Then following results holds true.

(i) For any $v \in \mathbf{W}^{2, q} \cap \mathbf{W}_{0}^{1, q}$, there is a constant $C=C(q, d(\Omega))$ such that

$$
\|v\|_{\mathbf{W}^{2, q}}:=\left\|\nabla^{2} v\right\|_{\mathbf{L}^{q}}+d(\Omega)^{-1}\|\nabla v\|_{\mathbf{L}^{q}}+d(\Omega)^{-2}\|v\|_{\mathbf{L}^{q}} \leq C\left\|\nabla^{2} v\right\|_{\mathbf{L}^{q}} .
$$

(ii) For $1<q<+\infty, f \in \mathbf{L}^{q}$, the Stokes problem

$$
-\Delta v+\nabla P=f, \text { in } \Omega,\left.\quad v\right|_{\Gamma}=0
$$

has a unique solution $(v, P)$ in $D\left(A_{q}\right) \times \mathbf{W}^{1, q}$. There exists a constant $C=C(q, d(\Omega))$ such that

$$
\left\|\nabla^{2} v\right\|_{\mathbf{L}^{q}}+\|\nabla P\|_{\mathbf{L}^{q}} \leq C\|f\|_{\mathbf{L}^{q}}
$$

Concerning the transport equation for the density function, we have the following result (that can be found e.g., in [8]).

Lemma 2.2. Let $v \in L^{1}(0, T ;$ Lip $)$ be a solenoidal vector field such that $v \cdot \mathbf{n}=0$ on $\Gamma$. For any $\rho_{0} \in W^{1, q}$ with $q \in[1,+\infty]$, the equation

$$
\rho_{t}+v \cdot \nabla \rho=0,\left.\quad \rho\right|_{t=0}=\rho_{0}(x)
$$

admits a unique solution $\rho \in L^{\infty}\left(0, T ; W^{1, \infty}\right) \cap C\left([0, T] ; \cap_{r<\infty} W^{1, r}\right)$ if $q=+\infty$ and $\rho \in$ $C\left([0, T] ; W^{1, q}\right)$ if $1 \leq q<+\infty$. Besides, the following estimate holds:

$$
\|\rho(t)\|_{W^{1, q}} \leq e^{\int_{0}^{t}\|\nabla v(\tau)\|_{\mathbf{L}} \infty d \tau}\left\|\rho_{0}\right\|_{W^{1, q}}, \quad \forall t \in[0, T] .
$$


If $\rho_{0} \in L^{p}$ for some $p \in[1,+\infty]$, then $\|\rho(t)\|_{L^{p}}=\left\|\rho_{0}\right\|_{L^{p}}$ for $t \in[0, T]$.

An essential characteristic of the director equation (for any given given velocity) is the following weak maximum principle (cf. [25]).

Lemma 2.3. Suppose $v \in L^{\infty}(0, T ; H) \cap L^{2}(0, T ; V), d_{0} \in \mathbf{H}^{1}(\Omega)$ with $d_{0} \in \mathbf{H}^{\frac{3}{2}}(\Gamma)$ and $\left|d_{0}\right| \leq 1$. If $d \in L^{\infty}\left(0, T ; \mathbf{H}^{1}\right) \cap L^{2}\left(0, T ; \mathbf{H}^{2}\right)$ is the weak solution of the initial boundary value problem

$$
\begin{aligned}
& d_{t}+v \cdot \nabla d=\gamma(\Delta d-f(d)), \quad \text { a.e. in } \Omega, \\
& \left.d\right|_{\Gamma}=d_{0}(x), \quad(x, t) \in \Gamma \times(0, T), \\
& \left.d\right|_{t=0}=d_{0}(x),
\end{aligned}
$$

then $|d(x, t)| \leq 1$, a.e. in $\Omega \times(0, T)$.

Finally, we report some inequalities that will be frequently used in the subsequent proof.

LEMMA 2.4. Let $\Omega$ be a bounded domain of $\mathbb{R}^{2}$ with smooth boundary.

(i) Ladyzhenskaya inequality. $\|v\|_{\mathbf{L}^{4}}^{2} \leq C\|\nabla v\|\|v\|, \quad \forall v \in \mathbf{H}_{0}^{1}(\Omega)$.

(ii) Agmon inequality. $\|f\|_{L^{\infty}}^{2} \leq C\|f\|_{H^{2}}\|f\|, \quad \forall f \in H^{2}(\Omega)$.

(iii) Poincaré inequality. For $1<q<+\infty,\|v\|_{\mathbf{L}^{q}} \leq C\|\nabla v\|_{\mathbf{L}^{q}}, \quad \forall v \in \mathbf{W}_{0}^{1, q}(\Omega)$.

(iv) Sobolev embeddings. For $1<q<+\infty$, the embedding $H^{1} \hookrightarrow L^{q}$ is compact. Besides, $\|f\|_{L^{\infty}} \leq C\|f\|_{W^{1, q}}, q>2, \quad \forall f \in W^{1, q}(\Omega)$.

The constant $C$ in the above inequalities may depend on $\Omega$ and $q$.

\section{Global strong solutions}

In order to prove the existence of strong solutions, we can first construct a sequence of approximate solutions $\left(\rho_{m}, v_{m}, d_{m}\right)$ within a semi-Galerkin scheme as in [7, Section 5] (we also refer to [25] for the case of homogeneous liquid crystal flow and [1] for the density-dependent Navier-Stokes equation with external forces). To prove the convergence of the approximate solutions, we only need to derive some a priori estimates for them. Due to the positivity condition (1.11), the Galerkin approximation in [7] does not rely on the introduction of a viscosity term in the transport equation (1.1), as in [14,33], and thus it can be used to establish (higher-order) Ladyzhenskaya type energy estimates. Since the calculations for the approximate solutions are (formally) identical to that as we work with smooth solutions, in what follows, we simply perform calculations for smooth solutions to problem (1.1)-(1.6).

3.1. Dissipative basic energy inequalities and lower-order energy estimates. The total energy of problem (1.1)-(1.6) is defined as follows:

$$
\mathcal{E}(t)=\frac{1}{2} \int_{\Omega} \rho(t)|v(t)|^{2} d x+\frac{\lambda}{2}\|\nabla d(t)\|^{2}+\lambda \int_{\Omega} F(d(t)) d x .
$$

In analogy to the constant density case (cf. [25]) or the nonhomogeneous system without external force (cf. [14,33]), our system (1.1)-(1.6) still has the following basic energy inequalities, which reflect the energy dissipation of the liquid crystal flow.

LEMma 3.1 (Basic energy inequalities). Let $(\rho, v, d)$ be a smooth solution of problem (1.1)-(1.6) on $\Omega \times[0, T]=Q_{T}(0 \leq T \leq+\infty)$.

(i) If $\mathbf{g}$ satisfies (F1), then

$$
\frac{d}{d t} \tilde{\mathcal{E}}(t)+\nu\|\nabla v(t)\|^{2}+\lambda \gamma\|\Delta d(t)-f(d(t))\|^{2}=0, \quad 0 \leq t \leq T,
$$


where

$$
\tilde{\mathcal{E}}(t)=\mathcal{E}(t)-\int_{\Omega} \rho \phi d x
$$

(ii) If $\mathbf{g}$ satisfies (F2), then

$$
\frac{d}{d t} \mathcal{E}(t)+\frac{\nu}{2}\|\nabla v(t)\|^{2}+\lambda \gamma\|\Delta d(t)-f(d(t))\|^{2} \leq \frac{C_{P}^{2} \bar{\rho}^{2}}{2 \nu}\|\mathbf{g}\|^{2}, \quad 0 \leq t \leq T .
$$

Proof. Multiplying (1.1), (1.2), and (1.4) by $\frac{1}{2}|v|^{2}, v$, and $\lambda(-\Delta d+f(d))$, respectively, and integrating over $\Omega$, we infer from the boundary conditions (1.6) and integration by parts that

$$
\begin{aligned}
& \frac{1}{2} \int_{\Omega} \rho_{t}|v|^{2} d x=-\frac{1}{2} \int_{\Omega} \nabla \cdot(\rho v)|v|^{2} d x=\frac{1}{2} \int_{\Omega} \rho v \cdot \nabla|v|^{2} d x \\
& \frac{1}{2} \int_{\Omega} \rho \frac{d}{d t}|v|^{2} d x+\nu\|\nabla v\|^{2} \\
&=-\frac{1}{2} \int_{\Omega} \rho v \cdot \nabla|v|^{2} d x+\int_{\Omega} \rho \mathbf{g} \cdot v d x-\lambda \int(\Delta d \cdot \nabla d) \cdot v d x, \\
& \frac{d}{d t}\left(\frac{\lambda}{2}\|\nabla d\|^{2}+\lambda \int_{\Omega} F(d) d x\right)+\lambda \gamma\|-\Delta f+f(d)\|^{2}+\lambda \int_{\Omega}(v \cdot \nabla d) \cdot \Delta d d x=0,
\end{aligned}
$$

where we have used the facts (cf. [25])

$$
\begin{aligned}
& \nabla \cdot(\nabla d \odot \nabla d)=\frac{1}{2} \nabla|\nabla d|^{2}+\Delta d \cdot \nabla d \\
& \int_{\Omega} \nabla P \cdot v d x=\int_{\Omega} \nabla|\nabla d|^{2} \cdot v d x=\int_{\Omega} v \cdot \nabla F(d) d x=0 .
\end{aligned}
$$

Adding (3.4)-(3.6) together, we can see that

$$
\frac{d}{d t} \mathcal{E}(t)+\nu\|\nabla v(t)\|^{2}+\lambda \gamma\|\Delta d(t)-f(d(t))\|^{2}=\int_{\Omega} \rho \mathbf{g} \cdot v d x .
$$

If $\mathbf{g}$ satisfies (F1) then, using the idea in [45], we multiply the transport equation (1.1) by $-\phi$ and integrate over $\Omega$ to get

$$
-\int_{\Omega} \rho_{t} \phi d x=\int_{\Omega} \nabla \cdot(\rho v) \phi d x=-\int_{\Omega} \rho \nabla \phi \cdot v d x
$$

Adding (3.9) with (3.10) and noticing that $\phi$ is independent of time, we arrive at our conclusion (3.2). On the other hand, if $\mathbf{g}$ satisfies (F2) then, using the Hölder inequality and Poincaré inequality, we infer that

$$
\left|\int_{\Omega} \rho \mathbf{g} \cdot v d x\right| \leq\|\rho\|_{L^{\infty}}\|\mathbf{g}\|\|v\| \leq C_{P} \bar{\rho}\|\mathbf{g}\|\|\nabla v\| \leq \frac{\nu}{2}\|\nabla v\|^{2}+\frac{C_{P}^{2} \bar{\rho}^{2}}{2 \nu}\|\mathbf{g}\|^{2},
$$

which together with (3.9) yields (3.3). 
Proposition 3.2. Under the assumptions of Theorem 1.1, the following estimates hold:

$$
\begin{aligned}
& 0<\underline{\rho} \leq \rho(x, t) \leq \bar{\rho}, \quad \forall t \geq 0 \\
& |d(x, t)| \leq 1, \quad \forall t \geq 0, \\
& \|v(t)\|+\|d(t)\|_{\mathbf{H}^{1}} \leq C, \quad \forall t \geq 0, \\
& \int_{0}^{+\infty}\left(\nu\|\nabla v(t)\|^{2}+\lambda \gamma\|\Delta d(t)-f(d(t))\|^{2}\right) d t \leq C,
\end{aligned}
$$

where $C$ is a constant depending on $\left\|v_{0}\right\|,\left\|d_{0}\right\|_{\mathbf{H}^{1}}, \eta, \underline{\rho}, \bar{\rho}, \Omega$, and also $\|\phi\|_{H^{1}}$ (under (F1)) or $\|\mathbf{g}\|_{L^{2}\left(0,+\infty ; \mathbf{L}^{2}\right)}$ (under (F2)).

Proof. (3.12) easily follows from the method of characteristics [18], while (3.13) is a consequence of the weak maximum principle for the director equation (see Lemma 2.3). If $\mathbf{g}$ satisfies (F1) then, since

$$
\left|\int_{\Omega} \rho \phi d x\right| \leq|\Omega| \bar{\rho}\|\phi\|_{L^{1}}<+\infty
$$

we can see that

$$
\tilde{\mathcal{E}}(t) \geq-|\Omega| \bar{\rho}\|\phi\|_{L^{1}}, \quad \forall t \geq 0 .
$$

The required uniform estimates follow from (3.2) and (3.16). If $\mathbf{g}$ satisfies (F2), by integrating (3.3) with respect to time, we arrive at the conclusion.

\subsection{Higher-order energy estimates. Denote}

$$
A(t)=\nu\|\nabla v(t)\|^{2}+\|\Delta d(t)-f(d(t))\|^{2} .
$$

LEMmA 3.3. The following inequality holds for smooth solutions $(\rho, v, d)$ to problem $(1.1)-(1.6)$ :

$$
\begin{aligned}
& \frac{d}{d t} A(t)+\left\|\rho^{\frac{1}{2}} v_{t}(t)\right\|^{2}+\gamma\|\nabla(\Delta d(t)-f(d(t)))\|^{2} \\
\leq & C\left(A^{2}(t)+A(t)\right)+C\|\mathbf{g}\|^{2}, \quad \forall t>0,
\end{aligned}
$$

where $C$ is a constant depending on $\left\|v_{0}\right\|,\left\|d_{0}\right\|_{\mathbf{H}^{1}}, \eta, \underline{\rho}, \bar{\rho}, \nu, \Omega$, and also $\|\phi\|_{H^{1}}$ (under (F1)) or $\|\mathbf{g}\|_{L^{2}\left(0,+\infty ; \mathbf{L}^{2}\right)}$ (under (F2)).

Proof. Using equations (1.1)-(1.4) and the facts (3.7), (3.8), we compute that

$$
\begin{aligned}
& \frac{\nu}{2} \frac{d}{d t}\|\nabla v\|^{2}=-\int_{\Omega} \nu \Delta v \cdot v_{t} d x \\
= & -\int_{\Omega} \rho\left|v_{t}\right|^{2} d x-\int_{\Omega} \rho(v \cdot \nabla v) \cdot v_{t} d x \\
& \quad-\lambda \int_{\Omega}[(\Delta d-f(d)) \cdot \nabla d] \cdot v_{t} d x+\int_{\Omega} \rho \mathbf{g} \cdot v_{t} d x
\end{aligned}
$$

and

$$
\frac{1}{2} \frac{d}{d t}\|\Delta d-f(d)\|^{2}
$$




$$
\begin{aligned}
& =\int_{\Omega}\left(\Delta d_{t}-f^{\prime}(d) d_{t}\right) \cdot(\Delta d-f(d)) d x \\
& =-\int \Delta(v \cdot \nabla d) \cdot(\Delta d-f(d)) d x-\gamma\|\nabla(\Delta d-f(d))\|^{2} \\
& \quad \quad-\int_{\Omega} f^{\prime}(d)[-v \cdot \nabla d+\gamma(\Delta d-f(d))](\Delta d-f(d)) d x \\
& =-\int(\Delta v \cdot \nabla d)(\Delta d-f(d)) d x-\int_{\Omega}\left[(v \cdot \nabla) \Delta d+2 \nabla v \nabla^{2} d\right] \cdot(\Delta d-f(d)) d x \\
& \quad-\gamma\|\nabla(\Delta d-f(d))\|^{2}-\gamma \int_{\Omega} f^{\prime}(d)(\Delta d-f(d)) \cdot(\Delta d-f(d)) d x .
\end{aligned}
$$

Adding (3.19) and (3.20) together, we have

$$
\begin{aligned}
& \frac{1}{2} \frac{d}{d t}\left(\nu\|\nabla v\|^{2}+\|\Delta d-f(d)\|^{2}\right)+\int_{\Omega} \rho\left|v_{t}\right|^{2} d x+\gamma\|\nabla(\Delta d-f(d))\|^{2} \\
=-\int_{\Omega} \rho(v \cdot \nabla v) \cdot v_{t} d x-\int_{\Omega}[(\Delta d-f(d)) \cdot \nabla d] \cdot\left(\Delta v+\lambda v_{t}\right) d x & \quad+\int_{\Omega} \rho \mathbf{g} \cdot v_{t} d x-\int_{\Omega}(v \cdot \nabla) \Delta d \cdot(\Delta d-f(d)) d x \\
& \quad-2 \int_{\Omega}\left(\nabla v \nabla^{2} d\right) \cdot(\Delta d-f(d)) d x \\
& -\gamma \int_{\Omega} f^{\prime}(d)(\Delta d-f(d)) \cdot(\Delta d-f(d)) d x \\
:= & \sum_{m=1}^{6} I_{m} .
\end{aligned}
$$

Next, we estimate $I_{1}, \ldots, I_{6}$ term by term. Using the fact (3.7), we rewrite (1.2) as

$$
-\nu \Delta v+\nabla\left(P+\frac{\lambda}{2}|\nabla d|^{2}+\lambda F(d)\right)=-\rho\left(v_{t}+v \cdot \nabla v\right)-\lambda(\Delta d-f(d)) \cdot \nabla d+\rho \mathbf{g} .
$$

By Lemma 2.1, we get

$$
\begin{aligned}
\|v\|_{\mathbf{H}^{2}} & \leq C\left(\left\|\rho v_{t}\right\|+\|\rho(v \cdot \nabla) v\|+\|(\Delta d-f(d)) \cdot \nabla d\|+\|\rho \mathbf{g}\|\right) \\
& \leq C\left(\bar{\rho}^{\frac{1}{2}}\left\|\rho^{\frac{1}{2}} v_{t}\right\|+\bar{\rho}\|v\|_{\mathbf{L}^{4}}\|\nabla v\|_{\mathbf{L}^{4}}+\bar{\rho}\|\mathbf{g}\|\right)+C\|(\Delta d-f(d)) \cdot \nabla d\| \\
& \leq C\left(\left\|\rho^{\frac{1}{2}} v_{t}\right\|+C\|v\|^{\frac{1}{2}}\|\nabla v\|\|v\|_{\mathbf{H}^{2}}^{\frac{1}{2}}+\|\mathbf{g}\|\right)+C\|(\Delta d-f(d)) \cdot \nabla d\| \\
& \leq \frac{1}{2}\|v\|_{\mathbf{H}^{2}}+C\left(\left\|\rho^{\frac{1}{2}} v_{t}\right\|+\|\mathbf{g}\|+\|(\Delta d-f(d)) \cdot \nabla d\|\right)+C\|\nabla v\|^{2},
\end{aligned}
$$

namely,

$$
\|v\|_{\mathbf{H}^{2}} \leq C\left(\left\|\rho^{\frac{1}{2}} v_{t}\right\|+\|\mathbf{g}\|+\|(\Delta d-f(d)) \cdot \nabla d\|\right)+C\|\nabla v\|^{2} .
$$

On the other hand, we have

$$
\begin{aligned}
& \|(\Delta d-f(d)) \cdot \nabla d\| \\
\leq & \|\nabla d\|_{\mathbf{L}^{4}}\|\Delta d-f(d)\|_{\mathbf{L}^{4}} \\
\leq & C\left(\|\Delta d\|^{\frac{1}{2}}\|\nabla d\|^{\frac{1}{2}}+\|\nabla d\|\right)\|\Delta d-f(d)\|^{\frac{1}{2}}\|\nabla(\Delta d-f(d))\|^{\frac{1}{2}}
\end{aligned}
$$




$$
\leq C\left(\|\Delta d-f(d)\|^{\frac{1}{2}}+1\right)\|\Delta d-f(d)\|^{\frac{1}{2}}\|\nabla(\Delta d-f(d))\|^{\frac{1}{2}} .
$$

Then we infer from (3.24), (3.25), and the Young inequality that

$$
\begin{aligned}
& I_{1} \leq \frac{1}{16} \int_{\Omega} \rho\left|v_{t}\right|^{2} d x+4 \int_{\Omega} \rho|v \cdot \nabla v|^{2} d x \\
& \leq \frac{1}{16} \int_{\Omega} \rho\left|v_{t}\right|^{2} d x+4 \bar{\rho}\|v\|_{\mathbf{L}^{4}}^{2}\|\nabla v\|_{\mathbf{L}^{4}}^{2} \\
& \leq \frac{1}{16} \int_{\Omega} \rho\left|v_{t}\right|^{2} d x+C\|v\|\|\nabla v\|\left(\|\nabla v\|\|\Delta v\|+\|\nabla v\|^{2}\right) \\
& \leq \frac{1}{16} \int_{\Omega} \rho\left|v_{t}\right|^{2} d x+C\|v\|_{\mathbf{H}^{2}}\|\nabla v\|^{2}+C\|\nabla v\|^{3} \\
& \leq \frac{1}{16} \int_{\Omega} \rho\left|v_{t}\right|^{2} d x+C\left\|\rho^{\frac{1}{2}} v_{t}\right\|\|\nabla v\|^{2}+C\left(\|\mathbf{g}\|+\|\nabla v\|^{2}\right)\|\nabla v\|^{2}+C\|\nabla v\|^{3} \\
& \quad+C\left(\|\Delta d-f(d)\|^{\frac{1}{2}}+1\right)\|\Delta d-f(d)\|^{\frac{1}{2}}\|\nabla(\Delta d-f(d))\|^{\frac{1}{2}}\|\nabla v\|^{2} \\
& \leq \frac{1}{8} \int_{\Omega} \rho\left|v_{t}\right|^{2} d x+\frac{\gamma}{8}\|\nabla(\Delta d-f(d))\|^{2}+C\|\nabla v\|^{2}+C\|\nabla v\|^{4} \\
& \quad+C\|\Delta d-f(d)\|^{2}+C\|\Delta d-f(d)\|^{4}+C\|\mathbf{g}\|^{2}, \\
& I_{2} \leq C\left(\|\Delta v\|+\underline{\rho}^{\frac{1}{2}}\left\|\rho^{\frac{1}{2}} v_{t}\right\|\right)\|(\Delta d-f(d)) \cdot \nabla d\| \\
& \quad \leq C\left(\left\|\rho^{\frac{1}{2}} v_{t}\right\|+\|\mathbf{g}\|+\|(\Delta d-f(d)) \cdot \nabla d\|+\|\nabla v\|^{2}\right)\|(\Delta d-f(d)) \cdot \nabla d\| \\
& \leq C\left(\left\|\rho^{\frac{1}{2}} v_{t}\right\|+\|\mathbf{g}\|+\|\nabla v\|^{2}\right) \\
& \quad \times\left(\|\Delta d-f(d)\|^{\frac{1}{2}}+1\right)\|\Delta d-f(d)\|^{\frac{1}{2}}\|\nabla(\Delta d-f(d))\|^{\frac{1}{2}} \\
& \quad+C\left(\|\Delta d-f(d)\|^{2}+\|\Delta d-f(d)\|\right)\|\nabla(\Delta d-f(d))\| \\
& \quad \leq \frac{1}{8} \int_{\Omega} \rho\left|v_{t}\right|^{2} d x+\frac{\gamma}{8}\|\nabla(\Delta d-f(d))\|^{2} \\
& \quad+C\|\Delta d-f(d)\|^{4}+C\|\Delta d-f(d)\|^{2}+C\|\nabla v\|^{4}+C\|\mathbf{g}\|^{2} .
\end{aligned}
$$

Concerning $I_{3}$, it follows that

$$
I_{3} \leq \bar{\rho}^{\frac{1}{2}}\|\mathbf{g}\|\left\|\rho^{\frac{1}{2}} v_{t}\right\| \leq \frac{1}{8}\left\|\rho v_{t}\right\|^{2}+2 \bar{\rho}\|\mathbf{g}\|^{2} .
$$

Next, using (3.14), we get

$$
\left\|f^{\prime}(d) \nabla d\right\| \leq C\left(\|d\|_{\mathbf{L}^{8}}^{2}+1\right)\|\nabla d\|_{\mathbf{L}^{4}} \leq C\left(1+\|\Delta d-f(d)\|^{\frac{1}{2}}\right),
$$

which implies that

$$
\begin{aligned}
I_{4} \leq C\|v\|_{\mathbf{L}^{4}}\|\nabla \Delta d\|\|\Delta d-f(d)\|_{\mathbf{L}^{4}} \\
\leq C\|v\|^{\frac{1}{2}}\|\nabla v\|^{\frac{1}{2}}\left(\|\nabla(\Delta d-f(d))\|+\left\|f^{\prime}(d) \nabla d\right\|\right)\|\Delta d-f(d)\|^{\frac{1}{2}}\|\nabla(\Delta d-f(d))\|^{\frac{1}{2}} \\
\leq C\|v\|^{\frac{1}{2}}\|\nabla v\|^{\frac{1}{2}}\|\Delta d-f(d)\|^{\frac{1}{2}}\|\nabla(\Delta d-f(d))\|^{\frac{3}{2}} \\
\quad+C\|v\|^{\frac{1}{2}}\|\nabla v\|^{\frac{1}{2}}\left(\|\Delta d-f(d)\|^{\frac{1}{2}}+\|\Delta d-f(d)\|\right)\|\nabla(\Delta d-f(d))\|^{\frac{1}{2}} \\
\quad \leq \frac{\gamma}{8}\|\nabla(\Delta d-f(d))\|^{2}+C\|\Delta d-f(d)\|^{4}+C\|\Delta d-f(d)\|^{2} \\
\quad+C\|\nabla v\|^{4}+C\|\nabla v\|^{2} .
\end{aligned}
$$


From the elliptic estimate and (3.14), we have

$$
\begin{aligned}
\|d\|_{\mathbf{H}^{2}} & \leq C\left(\|\Delta d\|+\|d\|_{\mathbf{H}^{\frac{3}{2}}(\Gamma)}\right) \\
& \leq C\left(\|\Delta d-f(d)\|+\|f(d)\|+\left\|d_{0}\right\|_{\mathbf{H}^{2}}\right. \\
& \leq C(\|\Delta d-f(d)\|+1) .
\end{aligned}
$$

It then follows from (3.24), (3.25), (3.26), and Young's inequality that

$$
\begin{gathered}
I_{5} \leq C\|\nabla v\|_{\mathbf{L}^{4}}\|d\|_{\mathbf{H}^{2}}\|\Delta d-f(d)\|_{\mathbf{L}^{4}} \\
\leq C\|\nabla v\|^{\frac{1}{2}}\|v\|_{\mathbf{H}^{2}}^{\frac{1}{2}}(\|\Delta d-f(d)\|+1)\|\Delta d-f(d)\|^{\frac{1}{2}}\|\nabla(\Delta d-f(d))\|^{\frac{1}{2}} \\
\leq C\|\nabla v\|^{\frac{1}{2}}\left(\left\|\rho^{\frac{1}{2}} v_{t}\right\|^{\frac{1}{2}}+\|\mathbf{g}\|^{\frac{1}{2}}+\|\nabla v\|\right)(\|\Delta d-f(d)\|+1) \\
\quad \times\|\Delta d-f(d)\|^{\frac{1}{2}}\|\nabla(\Delta d-f(d))\|^{\frac{1}{2}} \\
\quad+C\|\nabla v\|^{\frac{1}{2}}\left(\|\Delta d-f(d)\|^{\frac{5}{4}}+1\right)\|\Delta d-f(d)\|^{\frac{3}{4}}\|\nabla(\Delta d-f(d))\|^{\frac{3}{4}} \\
\leq \frac{1}{8} \int_{\Omega} \rho\left|v_{t}\right|^{2} d x+\frac{\gamma}{8}\|\nabla(\Delta d-f(d))\|^{2}+C\|\mathbf{g}\|^{2} \\
+C\|\nabla v\|^{4}+C\|\nabla v\|^{2}+C\|\Delta d-f(d)\|^{4}+C\|\Delta d-f(d)\|^{2} .
\end{gathered}
$$

Finally, for $I_{6}$, we have

$$
\begin{aligned}
I_{6} & \leq C\left\|f^{\prime}(d)\right\|_{\mathbf{L}^{4}}\|\Delta d-f(d)\|_{\mathbf{L}^{4}}\|\Delta d-f(d)\| \\
& \leq C\left(\|d\|_{\mathbf{L}^{8}}^{2}+1\right)\|\nabla(\Delta d-f(d))\|^{\frac{1}{2}}\|\Delta d-f(d)\|^{\frac{3}{2}} \\
& \leq \frac{\gamma}{8}\|\nabla(\Delta d-f(d))\|^{2}+C\|\Delta d-f(d)\|^{2} .
\end{aligned}
$$

Collecting the estimates for $I_{1}, \ldots, I_{6}$, we infer from (3.21) that

$$
\begin{gathered}
\frac{1}{2} \frac{d}{d t} A(t) \leq-\frac{1}{2} \int_{\Omega} \rho\left|v_{t}\right|^{2} d x-\frac{\gamma}{2}\|\nabla(\Delta d-f(d))\|^{2}+C\|\nabla v\|^{4}+C\|\nabla v\|^{2} \\
+C\|\Delta d-f(d)\|^{4}+C\|\Delta d-f(d)\|^{2}+C\|\mathbf{g}\|^{2}
\end{gathered}
$$

which yields our conclusion (3.18).

Proposition 3.4. Under the assumptions of Theorem 1.1, the following uniform estimates hold for any $t \geq 0$ :

$$
\begin{aligned}
& \|v(t)\|_{\mathbf{H}^{1}}+\|d(t)\|_{\mathbf{H}^{2}} \leq C, \\
& \sup _{t \geq 0} \int_{t}^{t+1}\left\|\rho^{\frac{1}{2}} v_{t}(\tau)\right\|^{2}+\|\nabla(\Delta d(\tau)-f(d(\tau)))\|^{2} d \tau \leq C,
\end{aligned}
$$

where $C$ is a constant depending on $\nu,\left\|v_{0}\right\|_{\mathbf{H}^{1}},\left\|d_{0}\right\|_{\mathbf{H}^{2}}, \eta, \underline{\rho}, \bar{\rho}$, and also $\|\phi\|_{H^{1}}$ (under (F1)) or $\|\mathbf{g}\|_{L^{2}\left(0,+\infty ; \mathbf{L}^{2}\right)}$ (under (F2)).

Proof. For both cases (F1) and (F2), (3.15) implies that

$$
\int_{0}^{+\infty} A(t) d t<+\infty
$$

The uniform bound (3.27) follows from Lemma 3.2, the higher-order energy inequality (3.18), and the uniform Gronwall lemma [38, Lemma III.1.1]. Integrating (3.18) from $t$ to $t+1$, we can conclude (3.28) from (3.27). 
Denote the quantity

$$
B(t)=\int_{\Omega} \rho\left|v_{t}(t)\right|^{2} d x+\left\|\nabla d_{t}(t)\right\|^{2}
$$

We can derive the following higher-order differential inequality.

LEMMA 3.5. If $\mathbf{g}$ satisfies (F1), then the following inequality holds for smooth solutions $(\rho, v, d)$ to problem $(1.1)-(1.6)$ :

$$
\frac{d}{d t} B(t)+\nu\left\|\nabla v_{t}\right\|^{2}+\gamma\left\|\Delta d_{t}\right\|^{2} \leq C\left(B^{2}(t)+B(t)\right)+C\|\nabla v\|^{2}, \quad \forall t \geq 0,
$$

where $C$ is a constant depending on $\nu,\left\|v_{0}\right\|_{\mathbf{H}^{1}},\left\|d_{0}\right\|_{\mathbf{H}^{2}}, \eta, \underline{\rho}, \bar{\rho}$, and $\|\phi\|_{H^{2}}$. On the other hand, if $\mathbf{g}$ satisfies (F2), then we have

$$
\begin{aligned}
& \frac{d}{d t} B(t)+\nu\left\|\nabla v_{t}\right\|^{2}+\gamma\left\|\Delta d_{t}\right\|^{2} \\
\leq & C\left(B^{2}(t)+B(t)\right)+C\left(\|\mathbf{g}\|_{\mathbf{H}^{1}}^{2}+\left\|\mathbf{g}_{t}\right\|^{2}+\|\nabla v\|^{2}\right), \quad \forall t \geq 0,
\end{aligned}
$$

where $C$ is a constant depending on $\nu,\left\|v_{0}\right\|_{\mathbf{H}^{1}},\left\|d_{0}\right\|_{\mathbf{H}^{2}}, \eta, \underline{\rho}, \bar{\rho}$, and $\|\mathbf{g}\|_{L^{2}\left(0,+\infty ; \mathbf{L}^{2}\right)}$.

Proof. Taking the temporal derivative of (1.2), computing the $L^{2}$ inner product of the resultant with $v_{t}$, and then using the fact $\nabla \cdot v=\nabla \cdot v_{t}=0$ and the transport equation (1.1), after integration by parts (keeping in mind that $\left.v\right|_{\Gamma}=\left.v_{t}\right|_{\Gamma}=0$ ), we get

$$
\begin{aligned}
& \frac{1}{2} \frac{d}{d t} \int_{\Omega} \rho\left|v_{t}\right|^{2} d x+\nu\left\|\nabla v_{t}\right\|^{2} \\
= & \frac{1}{2} \int_{\Omega} \rho_{t}\left|v_{t}\right|^{2} d x-\frac{1}{2} \int_{\Omega} \rho v \cdot \nabla\left(\left|v_{t}\right|^{2}\right) d x-\int_{\Omega} \rho\left(v_{t} \cdot \nabla\right) v \cdot v_{t} d x \\
& \quad-\int_{\Omega} \rho_{t}\left(v_{t}+v \cdot \nabla v\right) \cdot v_{t} d x-\int_{\Omega} \nabla P_{t} \cdot v_{t} d x+\int_{\Omega}\left(\rho_{t} \mathbf{g}+\rho \mathbf{g}_{t}\right) \cdot v_{t} d x \\
& \quad+2 \lambda \int_{\Omega}\left(\nabla d_{t} \odot \nabla d\right): \nabla v_{t} d x \\
=- & \int_{\Omega} \rho_{t}\left|v_{t}\right|^{2} d x-\int_{\Omega} \rho_{t}(v \cdot \nabla v) \cdot v_{t} d x-\int_{\Omega} \rho\left(v_{t} \cdot \nabla v\right) \cdot v_{t} d x \\
& \quad+\int_{\Omega}\left(\rho_{t} \mathbf{g}+\rho \mathbf{g}_{t}\right) \cdot v_{t} d x+2 \lambda \int_{\Omega}\left(\nabla d_{t} \odot \nabla d\right): \nabla v_{t} d x \\
=- & \int_{\Omega} v \cdot \nabla\left(\left|v_{t}\right|^{2}\right) d x-\int_{\Omega} \rho v \cdot \nabla\left[(v \cdot \nabla v) \cdot v_{t}\right] d x-\int_{\Omega} \rho\left(v_{t} \cdot \nabla v\right) \cdot v_{t} d x \\
& \quad+\int_{\Omega} \rho v \cdot \nabla\left(\mathbf{g} \cdot v_{t}\right) d x+\int_{\Omega} \rho \mathbf{g}_{t} \cdot v_{t} d x+2 \lambda \int_{\Omega}\left(\nabla d_{t} \odot \nabla d\right): \nabla v_{t} d x \\
:= & \sum_{m=1}^{6} J_{m} \cdot
\end{aligned}
$$

First, we consider the case that $\mathbf{g}$ satisfies the Assumption (F1). In this case, $J_{5}=0$. Using the uniform estimates in Proposition 3.4, we have

$$
\begin{aligned}
J_{1} & \leq C \bar{\rho}\left\|\nabla v_{t}\right\|\left\|v_{t}\right\|_{\mathbf{L}^{4}}\|v\|_{\mathbf{L}^{4}} \\
& \leq C\left\|\nabla v_{t}\right\|\left(\left\|\nabla v_{t}\right\|^{\frac{1}{2}}\left\|v_{t}\right\|^{\frac{1}{2}}+\left\|v_{t}\right\|\right)
\end{aligned}
$$




$$
\leq \frac{\nu}{12}\left\|\nabla v_{t}\right\|^{2}+C\left\|v_{t}\right\|^{2}
$$

Then for $J_{2}$, we infer from (3.24), (3.25), and (3.27) that

$$
\begin{aligned}
& J_{2} \leq \bar{\rho}\left(\|\nabla v\|_{\mathbf{L}^{4}}^{2}\|v\|_{\mathbf{L}^{4}}\left\|v_{t}\right\|_{\mathbf{L}^{4}}+\|v\|_{\mathbf{L}^{8}}^{2}\|v\|_{\mathbf{H}^{2}}\left\|v_{t}\right\|_{\mathbf{L}^{4}}+\|v\|_{\mathbf{L}^{8}}^{2}\|\nabla v\|_{\mathbf{L}^{4}}\left\|\nabla v_{t}\right\|\right) \\
& \leq C\left[\left(\|\Delta v\|\|\nabla v\|+\|\nabla v\|^{2}\right)\|\nabla v\|+\|v\|_{\mathbf{H}^{2}}\|\nabla v\|^{2}\right. \\
& \left.\quad+\|\nabla v\|^{2}\left(\|\Delta v\|^{\frac{1}{2}}\|\nabla v\|^{\frac{1}{2}}+\|\nabla v\|\right)\right]\left\|\nabla v_{t}\right\| \\
& \leq C\|\nabla v\|\left\|\nabla v_{t}\right\|+C\|v\|_{\mathbf{H}^{2}}\|\nabla v\|\left\|\nabla v_{t}\right\| \\
& \leq C\|\nabla v\|\left\|\nabla v_{t}\right\|+C\left(\left\|\rho^{\frac{1}{2}} v_{t}\right\|+\|\mathbf{g}\|+\|\nabla v\|^{2}\right)\|\nabla v\|\left\|\nabla v_{t}\right\| \\
& \quad+C\left(\|\Delta d-f(d)\|^{\frac{1}{2}}+1\right)\|\Delta d-f(d)\|^{\frac{1}{2}}\|\nabla(\Delta d-f(d))\|^{\frac{1}{2}}\|\nabla v\|\left\|\nabla v_{t}\right\| \\
& \leq \frac{\nu}{12}\left\|\nabla v_{t}\right\|^{2}+C\|\nabla(\Delta d-f(d))\|^{2}\|\nabla v\|^{2}+C\left\|\rho^{\frac{1}{2}} v_{t}\right\|^{2}\|\nabla v\|^{2} \\
& \quad+C\left(1+\|\mathbf{g}\|^{2}\right)\|\nabla v\|^{2} .
\end{aligned}
$$

When $\mathbf{g}=\nabla \phi$, then the last term on the right-hand side of (3.33) is controlled by $C\|\nabla v\|^{2}$. Next,

$$
\begin{aligned}
J_{3} & \leq \bar{\rho}\|\nabla v\|\left\|v_{t}\right\|_{\mathbf{L}^{4}}^{2} \leq C\left\|\nabla v_{t}\right\|\left\|v_{t}\right\| \leq \frac{\nu}{12}\left\|\nabla v_{t}\right\|^{2}+C\left\|v_{t}\right\|^{2}, \\
J_{4} & =\int_{\Omega} \rho v \cdot \nabla\left(\nabla \phi \cdot v_{t}\right) d x \\
& \leq \bar{\rho}\left(\|v\|_{\mathbf{L}^{4}}\left\|v_{t}\right\|_{\mathbf{L}^{4}}\|\phi\|_{H^{2}}+\left\|\nabla v_{t}\right\|\|v\|_{\mathbf{L}^{4}}\|\nabla \phi\|_{\mathbf{L}^{4}}\right) \\
& \leq C\|\nabla v\|\left\|\nabla v_{t}\right\| \\
& \leq \frac{\nu}{12}\left\|\nabla v_{t}\right\|^{2}+C\|\nabla v\|^{2} .
\end{aligned}
$$

Finally, for $J_{6}$, we have

$$
\begin{aligned}
J_{6} & \leq C\|\nabla d\|_{L^{\infty}}\left\|\nabla d_{t}\right\|\left\|\nabla v_{t}\right\| \\
& \leq C\|\nabla d\|_{H^{2}}\|\nabla d\|\left\|\nabla d_{t}\right\|\left\|\nabla v_{t}\right\| \\
& \leq C(\|\nabla(\Delta d-f(d))\|+1)\left\|\nabla d_{t}\right\|\left\|\nabla v_{t}\right\| \\
& \leq \frac{\nu}{12}\left\|\nabla v_{t}\right\|^{2}+C\left(\|\nabla(\Delta d-f(d))\|^{2}+1\right)\left\|\nabla d_{t}\right\|^{2} .
\end{aligned}
$$

Taking the temporal derivative of (1.4) and computing $L^{2}$ inner product of the resultant with $-\Delta d_{t}$, we obtain that

$$
\begin{aligned}
& \frac{1}{2} \frac{d}{d t}\left\|\nabla d_{t}\right\|^{2}+\gamma\left\|\Delta d_{t}\right\|^{2} \\
= & \int_{\Omega}\left(v_{t} \cdot \nabla d\right) \cdot \Delta d_{t} d x+\int_{\Omega}\left(v \cdot \nabla d_{t}\right) \cdot \Delta d_{t} d x+\gamma \int_{\Omega} f^{\prime}(d) d_{t} \cdot \Delta d_{t} d x \\
:= & J_{7}+J_{8}+J_{9},
\end{aligned}
$$

where the right-hand side of (3.34) can be estimated as follows:

$$
\begin{aligned}
J_{7} & \leq\left\|v_{t}\right\|_{\mathbf{L}^{4}}\|\nabla d\|_{\mathbf{L}^{4}}\left\|\Delta d_{t}\right\| \leq C\left\|\nabla v_{t}\right\|^{\frac{1}{2}}\left\|v_{t}\right\|^{\frac{1}{2}}\left\|\Delta d_{t}\right\| \\
& \leq \frac{\nu}{12}\left\|\nabla v_{t}\right\|^{2}+\frac{\gamma}{6}\left\|\Delta d_{t}\right\|+C\left\|v_{t}\right\|^{2}, \\
J_{8} & \leq\|v\|_{\mathbf{L}^{4}}\left\|\nabla d_{t}\right\|_{\mathbf{L}^{4}}\left\|\Delta d_{t}\right\| \leq C\left(\left\|\nabla d_{t}\right\|^{\frac{1}{2}}\left\|\Delta d_{t}\right\|^{\frac{1}{2}}+\left\|\nabla d_{t}\right\|\right)\left\|\Delta d_{t}\right\|
\end{aligned}
$$




$$
\begin{aligned}
& \leq \frac{\gamma}{6}\left\|\Delta d_{t}\right\|^{2}+C\left\|\nabla d_{t}\right\|^{2}, \\
J_{9} & \leq \gamma\left\|f^{\prime}(d)\right\|_{\mathbf{L} \infty}\left\|d_{t}\right\|\left\|\Delta d_{t}\right\| \leq \frac{\gamma}{6}\left\|\Delta d_{t}\right\|^{2}+C\left\|\nabla d_{t}\right\|^{2} .
\end{aligned}
$$

It follows from (1.4) that

$$
\nabla d_{t}=-\nabla(v \cdot \nabla d)+\gamma \nabla(\Delta d-f(d)) .
$$

Using the uniform estimate (3.27) and the Sobolev embedding theorem, we have

$$
\begin{aligned}
\|\nabla(v \cdot \nabla d)\| & \leq C\|\nabla v\|\|\nabla d\|_{\mathbf{L}^{\infty}}+C\|\|_{\mathbf{L}^{4}}\left\|\nabla^{2} d\right\|_{\mathbf{L}^{4}} \\
& \leq C\|\nabla v\|\|\nabla d\|_{\mathbf{H}^{2}}^{\frac{1}{2}}\|\nabla d\|^{\frac{1}{2}}+C\|\nabla v\|\left(\|\nabla \Delta d\|^{\frac{1}{2}}\|\Delta d\|^{\frac{1}{2}}+\|\Delta d\|\right) \\
& \leq C\|\nabla v\|\|\nabla \Delta d\|^{\frac{1}{2}}+C\|\nabla v\| \\
& \leq C\|\nabla v\|\|\nabla(\Delta d-f(d))\|^{\frac{1}{2}}+C\|\nabla v\|,
\end{aligned}
$$

which together with the equality (3.35) implies that

$$
\begin{aligned}
\|\nabla(\Delta d-f(d))\| & \leq \frac{1}{\gamma}\left\|\nabla d_{t}\right\|+\frac{1}{\gamma}\|\nabla(v \cdot \nabla d)\| \\
& \leq \frac{1}{\gamma}\left\|\nabla d_{t}\right\|+C\|\nabla v\|\|\nabla(\Delta d-f(d))\|^{\frac{1}{2}}+C\|\nabla v\| \\
& \leq \frac{1}{\gamma}\left\|\nabla d_{t}\right\|+\frac{1}{2}\|\nabla(\Delta d-f(d))\|+C\|\nabla v\| .
\end{aligned}
$$

As a result, we get

$$
\begin{aligned}
\left\|\nabla d_{t}\right\| & \leq\|\nabla(v \cdot \nabla d)\|+\gamma\|\nabla(\Delta d-f(d))\| \\
& \leq C\|\nabla v\|\|\nabla(\Delta d-f(d))\|^{\frac{1}{2}}+C\|\nabla v\|+\gamma\|\nabla(\Delta d-f(d))\| \\
& \leq(1+\gamma)\|\nabla(\Delta d-f(d))\|+C\|\nabla v\| .
\end{aligned}
$$

Collecting the above estimates for $J_{1}, \ldots, J_{9}$ and using the relations $(3.37),(3.38)$, we conclude from (3.32) and (3.34) that

$$
\begin{aligned}
& \frac{d}{d t} B(t)+\nu\left\|\nabla v_{t}\right\|^{2}+\gamma\left\|\Delta d_{t}\right\|^{2} \\
\leq & C\|\nabla v\|^{2}\left(\left\|\rho^{\frac{1}{2}} v_{t}\right\|^{2}+\left\|\nabla d_{t}\right\|^{2}\right)+C\left\|\nabla d_{t}\right\|^{4}+C\left(\left\|v_{t}\right\|^{2}+\left\|\nabla d_{t}\right\|^{2}\right)+C\|\nabla v\|^{2},
\end{aligned}
$$

which yields our conclusion (3.30).

Now if $\mathbf{g}$ satisfies (F2), we only have to re-estimate the terms $J_{2}, J_{4}$, and $J_{5}$ using the uniform estimate (3.27). It follows from (3.33) that

$$
\begin{aligned}
J_{2} & \leq \frac{\nu}{12}\left\|\nabla v_{t}\right\|^{2}+C\|\nabla(\Delta d-f(d))\|^{2}\|\nabla v\|^{2}+C\left\|\rho^{\frac{1}{2}} v_{t}\right\|^{2}\|\nabla v\|^{2}+C\left(1+\|\mathbf{g}\|^{2}\right)\|\nabla v\|^{2} \\
& \leq \frac{\nu}{12}\left\|\nabla v_{t}\right\|^{2}+C\|\nabla(\Delta d-f(d))\|^{2}\|\nabla v\|^{2}+C\left\|\rho^{\frac{1}{2}} v_{t}\right\|^{2}\|\nabla v\|^{2}+C\|\mathbf{g}\|^{2}+C\|\nabla v\|^{2} .
\end{aligned}
$$

Moreover,

$$
\begin{aligned}
J_{4} & \leq \bar{\rho}\left(\|v\|_{\mathbf{L}^{4}}\left\|v_{t}\right\|_{\mathbf{L}^{4}}\|\mathbf{g}\|_{\mathbf{H}^{1}}+\left\|\nabla v_{t}\right\|\|v\|_{\mathbf{L}^{4}}\|\mathbf{g}\|_{\mathbf{L}^{4}}\right) \\
& \leq C\|\mathbf{g}\|_{\mathbf{H}^{1}}\|\| \nabla v\|\| \nabla v_{t} \|
\end{aligned}
$$




$$
\begin{aligned}
& \leq \frac{\nu}{24}\left\|\nabla v_{t}\right\|^{2}+C\|\mathbf{g}\|_{\mathbf{H}^{1}}^{2}, \\
J_{5} & \leq \bar{\rho}\left\|\mathbf{g}_{t}\right\|\left\|v_{t}\right\| \leq \frac{\nu}{24}\left\|\nabla v_{t}\right\|^{2}+C\left\|\mathbf{g}_{t}\right\|^{2} .
\end{aligned}
$$

By the above estimates, we easily arrive at (3.31).

Proposition 3.6. Under the assumptions of Theorem 1.1, the following uniform estimates hold for any $t \geq 0$ :

$$
\begin{aligned}
& \left\|v_{t}(t)\right\|+\left\|d_{t}(t)\right\|_{\mathbf{H}^{1}} \leq C, \\
& \|v(t)\|_{\mathbf{H}^{2}}+\|d(t)\|_{\mathbf{H}^{3}} \leq C, \\
& \sup _{t \geq 0} \int_{t}^{t+1}\left(\left\|\nabla v_{t}(\tau)\right\|^{2}+\left\|\Delta d_{t}(\tau)\right\|^{2}\right) d \tau \leq C, \\
& \sup _{t \geq 0} \int_{t}^{t+1}\|v(\tau)\|_{\mathbf{W}^{2, q}}^{2} d \tau \leq C, \quad \forall q \in(1,+\infty) .
\end{aligned}
$$

Moreover, for any $T>0$,

$$
\|\rho(t)\|_{W^{1, q}} \leq e^{C T}\left\|\rho_{0}\right\|_{W^{1, q}}, \quad \forall t \in[0, T] .
$$

The constant $C$ in the above estimates depends on $\nu,\left\|v_{0}\right\|_{\mathbf{H}^{2}},\left\|d_{0}\right\|_{\mathbf{H}^{3}}, \eta, \rho, \bar{\rho}$, and also $\|\phi\|_{H^{2}}$ (under (F1)) or $\|\mathbf{g}\|_{L^{2}\left(0,+\infty ; \mathbf{H}^{1}\right)},\left\|\mathbf{g}_{t}\right\|_{L^{2}\left(0,+\infty ; \mathbf{L}^{2}\right)}$ (under (F2)), but it is independent of $t$.

Proof. It follows from (3.28), the definition of $B(t)$, and (3.38) that

$$
\sup _{t \geq 0} \int_{t}^{t+1} B(\tau) d \tau \leq C
$$

where $C$ is a constant depending on $\nu,\left\|v_{0}\right\|_{\mathbf{H}^{1}},\left\|d_{0}\right\|_{\mathbf{H}^{2}}, \eta, \underline{\rho}, \bar{\rho}$, and also $\|\phi\|_{H^{1}}$ (under $(\mathrm{F} 1))$ or $\|\mathbf{g}\|_{L^{2}\left(0,+\infty ; \mathbf{L}^{2}\right)}$ (under (F2)). Applying the uniform Gronwall lemma again, we infer from (3.30) (or (3.31)) and (3.44) that

$$
B(t) \leq C, \quad \forall t \geq 0,
$$

where $C$ is a constant depending on $\nu,\left\|v_{0}\right\|_{\mathbf{H}^{2}},\left\|d_{0}\right\|_{\mathbf{H}^{3}}, \eta, \rho, \bar{\rho}$, and also $\|\phi\|_{H^{2}}$ (under (F1)) or $\|\mathbf{g}\|_{L^{2}\left(0,+\infty ; \mathbf{H}^{1}\right)},\left\|\mathbf{g}_{t}\right\|_{L^{2}\left(0,+\infty ; \mathbf{L}^{2}\right)}$ (under (F2)). This yields the estimate (3.39). Integrating (3.30) or (3.31) from $t$ to $t+1$, we conclude (3.41). Recalling (3.22), we deduce from Lemma 2.1 and the estimates (3.27), (3.37) that

$$
\begin{aligned}
\|v\|_{\mathbf{H}^{2}} & \leq C\left(\left\|\rho v_{t}\right\|+\|\rho(v \cdot \nabla v)\|+\|(\Delta d-f(d)) \cdot \nabla d\|+\|\rho \mathbf{g}\|\right) \\
& \leq C \bar{\rho}\left(\left\|v_{t}\right\|+\|v\|_{\mathbf{L}^{4}}\|\nabla v\|_{\mathbf{L}^{4}}+\|\mathbf{g}\|\right)+C\|\nabla d\|_{\mathbf{L}^{4}}\|\Delta d-f(d)\|_{\mathbf{L}^{4}} \\
& \leq C\left(\left\|v_{t}\right\|+\|\nabla v\|\|v\|_{\mathbf{H}^{2}}^{\frac{1}{2}}\|\nabla v\|^{\frac{1}{2}}+\|\mathbf{g}\|\right) \\
& \quad+C\|d\|_{\mathbf{H}^{2}}\left(\|\nabla(\Delta d-f(d))\|^{\frac{1}{2}}\|\Delta d-f(d)\|^{\frac{1}{2}}\right) \\
& \leq \frac{1}{2}\|v\|_{\mathbf{H}^{2}}+C\left(\left\|v_{t}\right\|+\left\|\nabla d_{t}\right\|+\|\nabla v\|+\|\mathbf{g}\|\right) .
\end{aligned}
$$

Using (3.39) and the facts that either under (F1) $\|\mathbf{g}\| \leq\|\phi\|_{H^{1}}$, or under (F2) $\|\mathbf{g}\|$ is bounded by a constant depending on $\|\mathbf{g}\|_{L^{2}\left(0,+\infty ; \mathbf{L}^{2}\right)}$ and $\left\|\mathbf{g}_{t}\right\|_{L^{2}\left(0,+\infty ; \mathbf{L}^{2}\right)}$, we get the uniform estimate for $\|v\|_{\mathbf{H}^{2}}$. 
Next, by the elliptic estimate, we infer from (3.35) that

$$
\begin{aligned}
\|\nabla d\|_{\mathbf{H}^{2}} & \leq C\left(\left\|\nabla d_{t}\right\|+\|\nabla(v \cdot \nabla d)\|+\left\|f^{\prime}(d) \nabla d\right\|+\left\|\nabla d_{0}\right\|_{\mathbf{H}^{\frac{3}{2}}(\Gamma)}+\|\nabla d\|\right) \\
& \leq C+C\|\nabla v\|_{\mathbf{L}^{4}}\|\nabla d\|_{\mathbf{L}^{4}}+C\|v\|_{\mathbf{L}^{\infty}}\|d\|_{\mathbf{H}^{2}} \\
& \leq C+C\|v\|_{\mathbf{H}^{2}}\|d\|_{\mathbf{H}^{2}} .
\end{aligned}
$$

Combining (3.46) and (3.47), we obtain the uniform estimate (3.40). In a similar manner to (3.46), for $q \in(1,+\infty)$, using (3.40), we get

$$
\begin{aligned}
\|v\|_{\mathbf{W}^{2, q}} & \leq C\left(\left\|\rho v_{t}\right\|_{\mathbf{L}^{q}}+\|\rho(v \cdot \nabla v)\|_{\mathbf{L}^{q}}+\|(\Delta d-f(d)) \cdot \nabla d\|_{\mathbf{L}^{q}}+\|\rho \mathbf{g}\|_{\mathbf{L}^{q}}\right) \\
& \leq C \bar{\rho}\left(\left\|v_{t}\right\|_{\mathbf{L}^{q}}+\|v\|_{\mathbf{L}^{\frac{q}{2}}}\|\nabla v\|_{\mathbf{L}^{\frac{q}{2}}}+\|\mathbf{g}\|_{\mathbf{L}^{q}}\right)+C\|\nabla d\|_{\mathbf{L}^{\frac{q}{2}}}\|\Delta d-f(d)\|_{\mathbf{L}^{\frac{q}{2}}} \\
& \leq C\left(\left\|\nabla v_{t}\right\|+\|v\|_{\mathbf{H}^{2}}+\|\nabla(\Delta d-f(d))\|+\|\mathbf{g}\|_{\mathbf{L}^{q}}\right) \\
& \leq C\left(\left\|\nabla v_{t}\right\|+\|\nabla(\Delta d-f(d))\|+\|\mathbf{g}\|_{\mathbf{H}^{1}}+\|\nabla v\|\right)
\end{aligned}
$$

Thus, (3.42) is a consequence of (3.48), (3.28), and (3.41), where the bound either depends on $\|\phi\|_{H^{2}}($ under $(\mathrm{F} 1))$ or $\|\mathbf{g}\|_{L^{2}\left(0,+\infty ; \mathbf{H}^{1}\right)}$ (under (F2)). Finally, for any $T>0$,

$$
\begin{aligned}
\int_{0}^{t}\|\nabla v(\tau)\|_{\mathbf{L}^{\infty}} d \tau & \leq C \int_{0}^{T}\|v(\tau)\|_{\mathbf{W}^{2, r}} d \tau \\
& \leq C T^{\frac{1}{2}}\left(\int_{0}^{T}\|v(\tau)\|_{\mathbf{W}^{2, r}}^{2} d \tau\right)^{\frac{1}{2}} \\
& \leq C T, \quad \forall t \in[0, T], \text { for some } r>2 .
\end{aligned}
$$

Then we infer from (2.1) that (3.43) holds. The proof is complete.

\subsection{Proof of Theorem 1.1.}

Proof. Under the assumptions in Theorem 1.1, we can derive estimates for the approximate solutions to problem (1.1)-(1.6) $\left(\rho_{m}, v_{m}, d_{m}\right)$ as in Propositions 3.2, 3.4, and 3.6, which are independent of the parameter $m$. By extracting a subsequence and passing to limit as $m \rightarrow+\infty$, we can obtain a global strong solution $(\rho, v, d)$ to problem (1.1)-(1.6) in a standard way, such that

$$
\begin{aligned}
& \rho \in L^{\infty}\left([0, T], W^{1, r}(\Omega)\right), \\
& v \in L^{\infty}\left([0, T] ; \mathbf{H}^{2}(\Omega) \cap V\right) \cap L^{2}\left(0, T ; \mathbf{W}^{2, q}\right), \quad v_{t} \in L^{2}(0, T ; \mathbf{V}), q \in(1,+\infty), \\
& d \in L^{\infty}\left([0, T] ; \mathbf{H}^{3}(\Omega)\right), \quad d_{t} \in L^{\infty}\left([0, T] ; \mathbf{H}_{0}^{1}(\Omega)\right) \cap L^{2}\left(0, T ; \mathbf{H}^{2}\right), \\
& 0<\underline{\rho} \leq \rho(x, t) \leq \bar{\rho}, \quad|d(x, t)| \leq 1, \quad \forall(x, t) \in \Omega \times[0, T] .
\end{aligned}
$$

Due to Lemma 2.2, we infer that $\rho \in C\left([0, T], W^{1, r}(\Omega)\right)$. It is not difficult to see from (3.40) and (3.41) that $d \in L^{2}\left(0, T ; \mathbf{H}^{4}\right)$, which together with $d_{t} \in L^{2}\left(0, T ; \mathbf{H}^{2}\right)$ yields that $d \in C\left([0, T] ; \mathbf{H}^{3}\right)$. By the regularity of the Stokes operator,

$$
\begin{aligned}
&\|v\|_{\mathbf{H}^{3}} \leq C\left(\left\|\rho v_{t}\right\|_{\mathbf{H}^{1}}+\|\rho(v \cdot \nabla v)\|_{\mathbf{H}^{1}}+\|(\Delta d-f(d)) \cdot \nabla d\|_{\mathbf{H}^{1}}+\|\rho \mathbf{g}\|_{\mathbf{H}^{1}}\right) \\
& \leq C \bar{\rho}\left(\left\|v_{t}\right\|_{\mathbf{H}^{1}}+\|v \cdot \nabla v\|_{\mathbf{H}^{1}}+\|\mathbf{g}\|_{\mathbf{H}^{1}}\right) \\
& \quad+C\|\nabla \rho\|_{\mathbf{L}^{q^{\prime}}}\left(\left\|v_{t}\right\|_{\mathbf{L}^{q}}+\|v \cdot \nabla v\|_{\mathbf{L}^{q}}+\|\mathbf{g}\|_{\mathbf{L}^{q}}\right)+C\|\nabla d\|_{\mathbf{L}^{\infty}}\|\Delta d-f(d)\|_{\mathbf{H}^{1}} \\
& \quad+C\left\|\nabla^{2} d\right\|_{\mathbf{L}^{q^{\prime}}}\|\Delta d-f(d)\|_{\mathbf{L}^{q}}, \text { for some } 2<q, q^{\prime}<+\infty, \frac{1}{q}+\frac{1}{q^{\prime}}=\frac{1}{2}
\end{aligned}
$$


and estimates (3.40), (3.41), (3.43), we infer that $v \in L^{2}\left(0, T ; \mathbf{H}^{3}\right)$. This fact and $v_{t} \in L^{2}(0, T ; \mathbf{V})$ yields the continuity $v \in C\left([0, T], \mathbf{H}^{2}\right)$.

Finally, we briefly show that the strong solution is indeed unique. Suppose $(\rho, v, d)$ and $(\tilde{\rho}, \tilde{v}, \tilde{d})$ are two strong solutions corresponding to the same initial data $\left(\rho_{0}, v_{0}, d_{0}\right)$. One can easily check that all the computations in [7, Section 4] can be verified due to the regularity of the two strong solutions. Denote $\delta \rho=\rho-\tilde{\rho}, \delta v=v-\tilde{v}$, and $\delta d=d-\tilde{d}$. We have, for any $t \in[0, T]$ (cf. e.g., [7, (4.46)]),

$$
\begin{aligned}
& \frac{1}{2}\|\delta \rho(t)\|^{2}+\frac{1}{2} \int_{\Omega} \tilde{\rho}(t)|\delta v(t)|^{2} d x+\frac{1}{2}\|\nabla(\delta d(t))\|^{2} \\
\leq-\int_{0}^{t} & \|\nabla(\delta v)\|^{2} d t-\int_{0}^{t}\|\Delta(\delta d)\|^{2} d t+\int_{0}^{t} \int_{\Omega}(\delta \rho)(\delta v) \nabla \rho d x d t \\
& -\int_{0}^{t} \int_{\Omega} \tilde{\rho} \nabla v|\delta v|^{2} d x d t+\int_{0}^{t} \int_{\Omega}(\delta \rho)(\delta v)\left(v_{t}+v \cdot \nabla v\right) d x d t \\
& +\int_{0}^{t} \int_{\Omega} v(\delta d) \Delta(\delta d) d x d t-\int_{0}^{t} \int_{\Omega}(\delta v) \nabla(\delta d) \Delta d d x d t \\
& +\int_{0}^{t} \int_{\Omega} \Delta(\delta d)(f(d)-f(\tilde{d})) d x d t .
\end{aligned}
$$

Using the Hölder inequality, Young inequality, and the estimates (3.39), (3.40), (3.43), we easily get

$$
\|\delta \rho(t)\|^{2}+\int_{\Omega} \tilde{\rho}(t)|\delta v(t)|^{2} d x+\|\nabla(\delta d(t))\|^{2} \leq C_{T} \int_{0}^{t}\left(\|\delta \rho\|^{2}+\|\delta v\|^{2}+\|\nabla(\delta d)\|^{2}\right) d t .
$$

Due to the positivity of the density $\tilde{\rho} \geq \rho>0$, the uniqueness follows from the above estimate and the Gronwall inequality. The proof is complete.

\section{Long-time behavior}

In this section, we study the long-time behavior of global strong solutions to problem (1.1)-(1.6).

Proposition 4.1. Under the assumptions in Theorem 1.1 (either $\mathbf{g}$ satisfies (F1) or (F2)), the global strong solution to (1.1)-(1.6) has the following decay property:

$$
\lim _{t \rightarrow+\infty}\left(\|v(t)\|_{\mathbf{H}^{1}}+\|\Delta d(t)-f(d(t))\|\right)=0 .
$$

Proof. For both cases (F1) and (F2), (3.15) implies that $\int_{0}^{+\infty} A(t) d t<+\infty$. It then follows from (3.18) and [46, Lemma 6.2.1] that $\lim _{t \rightarrow+\infty} A(t)=0$, which together with the Poincaré inequality yields (4.1).

Proposition 4.2. Under the assumptions in Theorem 1.1 (either $\mathbf{g}$ satisfies (F1) or (F2)), the global strong solution to (1.1)-(1.6) has the following decay property:

$$
\lim _{t \rightarrow+\infty}\left(\left\|v_{t}(t)\right\|_{\mathbf{H}^{1}}+\|\nabla(\Delta d(t)-f(d(t)))\|+\left\|d_{t}\right\|_{\mathbf{H}^{1}}\right)=0 .
$$

Proof. First, we look at the case that $\mathbf{g}$ satisfies (F2). We have already proved that $A(t)$ is bounded for all time and $A(t) \in L^{1}(0,+\infty)$. Therefore, integrating (3.18) from 0 to $+\infty$ with respect to time, we infer from (F2) that

$$
\int_{0}^{+\infty}\left(\left\|\rho^{\frac{1}{2}} v_{t}(t)\right\|^{2}+\gamma\|\nabla(\Delta d(t)-f(d(t)))\|^{2}\right) d t
$$




$$
\begin{aligned}
& \leq A(0)+C\left(\sup _{t \geq 0} A(t)+1\right) \int_{0}^{+\infty} A(t) d t+C \int_{0}^{+\infty}\|\mathbf{g}(t)\|^{2} d t \\
& <+\infty .
\end{aligned}
$$

Recalling (3.38), we obtain

$$
\int_{0}^{+\infty} B(t) d t<+\infty
$$

Then using (3.31), Assumption (F2), and [46, Lemma 6.2.1], we conclude that

$$
\lim _{t \rightarrow+\infty} B(t)=0,
$$

which combined with Poincaré inequality and (3.37), (3.38) yields (4.2).

Next, we deal with the case when $\mathbf{g}$ satisfies (F1). We only need to show that (4.4) still holds in this case. For this purpose, we re-estimate the terms $I_{2}, I_{3}, I_{4}$, and $I_{5}$ in (3.21) using the higher-order estimate (3.40) instead of the lower-order one (3.27). As in [45], we infer from the transport equation (1.1) and integration by parts that

$$
\begin{aligned}
I_{3} & =\frac{d}{d t} \int_{\Omega} \rho \nabla \phi \cdot v d x-\int_{\Omega} \rho_{t} \nabla \phi \cdot v d x \\
& =\frac{d}{d t} \int_{\Omega} \rho \nabla \phi \cdot v d x-\int_{\Omega} \rho v \cdot \nabla(\nabla \phi \cdot v) d x \\
& \leq \frac{d}{d t} \int_{\Omega} \rho \nabla \phi \cdot v d x+\bar{\rho}\left(\|v\|_{\mathbf{L}^{4}}^{2}\|\phi\|_{H^{2}}+\|v\|_{\mathbf{L}^{4}}\|\nabla \phi\|_{\mathbf{L}^{4}}\|\nabla v\|\right) \\
& \leq \frac{d}{d t} \int_{\Omega} \rho \nabla \phi \cdot v d x+C\left(\|v\|\|\nabla v\|+\|v\|^{\frac{1}{2}}\|\nabla v\|^{\frac{3}{2}}\right) \\
& \leq \frac{d}{d t} \int_{\Omega} \rho \nabla \phi \cdot v d x+C\|\nabla v\|^{2} .
\end{aligned}
$$

Next, since $\Delta d-\left.f(d)\right|_{\Gamma}=0$, we obtain that

$$
\begin{aligned}
I_{2}+I_{4}+I_{5}= & -\lambda \int_{\Omega}[(\Delta d-f(d)) \cdot \nabla d] \cdot v_{t} d x-\int_{\Omega} \Delta(v \cdot \nabla d) \cdot(\Delta d-f(d)) d x \\
= & -\lambda \int_{\Omega}[(\Delta d-f(d)) \cdot \nabla d] \cdot v_{t} d x+\int_{\Omega} \nabla(v \cdot \nabla d) \cdot \nabla(\Delta d-f(d)) d x \\
= & -\lambda \int_{\Omega}[(\Delta d-f(d)) \cdot \nabla d] \cdot v_{t} d x+\int_{\Omega} \nabla_{k} v_{i} \nabla_{i} d_{j} \nabla_{k}\left(\Delta d_{j}-f_{j}(d)\right) d x \\
& \quad+\int_{\Omega} v_{i} \nabla_{k} \nabla_{i} d_{j} \nabla_{k}\left(\Delta d_{j}-f_{j}(d)\right) d x \\
:= & I_{2}^{\prime}+I_{4}^{\prime}+I_{5}^{\prime}
\end{aligned}
$$

where

$$
\begin{aligned}
I_{2}^{\prime} & \leq C \underline{\rho}^{\frac{1}{2}}\left\|\rho^{\frac{1}{2}} v_{t}\right\|\|(\Delta d-f(d)) \cdot \nabla d\| \\
& \leq C\left\|\rho^{\frac{1}{2}} v_{t}\right\|\left(\|\Delta d-f(d)\|^{\frac{1}{2}}+1\right)\|\Delta d-f(d)\|^{\frac{1}{2}}\|\nabla(\Delta d-f(d))\|^{\frac{1}{2}} \\
& \leq \frac{3}{8} \int_{\Omega} \rho\left|v_{t}\right|^{2} d x+\frac{\gamma}{8}\|\nabla(\Delta d-f(d))\|^{2}+C\|\Delta d-f(d)\|^{4}+C\|\Delta d-f(d)\|^{2},
\end{aligned}
$$




$$
\begin{aligned}
I_{4}^{\prime}+I_{5}^{\prime} & \leq\|\nabla v\|\|\nabla d\|_{\mathbf{L}^{\infty}}\|\nabla(\Delta d-f(d))\|+\|v\|_{\mathbf{L}^{4}}\left\|\nabla^{2} d\right\|_{\mathbf{L}^{4}}\|\nabla(\Delta d-f(d))\| \\
& \leq C\|\nabla v\|\|d\|_{\mathbf{H}^{3}}\|\nabla(\Delta d-f(d))\| \\
& \leq \frac{\gamma}{4}\|\nabla(\Delta d-f(d))\|^{2}+C\|\nabla v\|^{2} .
\end{aligned}
$$

Replacing the original estimates for $I_{2}, \ldots, I_{5}$ by $I_{2}^{\prime}, \ldots, I_{5}^{\prime}$, we arrive at the following inequality:

$$
\begin{aligned}
& \frac{d}{d t}\left(A(t)-2 \int_{\Omega} \rho \nabla \phi \cdot v d x\right)+\left\|\rho^{\frac{1}{2}} v_{t}(t)\right\|^{2}+\gamma\|\nabla(\Delta d(t)-f(d(t)))\|^{2} \\
\leq & C\left(A^{2}(t)+A(t)\right)
\end{aligned}
$$

where $C$ is a constant depending on $\left\|v_{0}\right\|,\left\|d_{0}\right\|_{\mathbf{H}^{1}}, \eta, \underline{\rho}, \bar{\rho}, \nu, \Omega$, and also $\|\phi\|_{H^{2}}$. Integrating (4.7) with respect to time from 0 to $+\infty$, we deduce that

$$
\begin{aligned}
& \int_{0}^{+\infty}\left\|\rho^{\frac{1}{2}} v_{t}(t)\right\|^{2}+\gamma\|\nabla(\Delta d(t)-f(d(t)))\|^{2} d t \\
\leq & A(0)-2 \int_{\Omega} \rho_{0} \nabla \phi \cdot v_{0} d x+2\left|\int_{\Omega} \rho \nabla \phi v d x\right| \\
& +C\left(\sup _{t \geq 0} A(t)+1\right) \int_{0}^{+\infty} A(t) d t \\
<+\infty &
\end{aligned}
$$

which again implies (4.4). Using the same argument as for the previous case, we conclude the decay property (4.2). The proof is complete.

\subsection{Proof of Theorem 1.2.}

Proof. According to Propositions 4.1 and 4.2, it remains to show the convergence for the density function $\rho$ and the director vector $d$.

As a direct consequence of the uniform-in-time estimate (3.12) and weak compactness of bounded sets in $L^{q}(1<q<+\infty)$, we know that for any sequence $\left\{t_{i}\right\} \nearrow+\infty$, there is a subsequence $\left\{t_{i}^{\prime}\right\} \nearrow+\infty$ such that $\rho\left(t_{i}^{\prime}\right)$ converges weakly to a certain $\rho_{\infty}$ in $L^{q}$. On the other hand, due to Lemma 2.2, $\left\|\rho\left(t_{i}^{\prime}\right)\right\|_{L^{q}}=\left\|\rho_{\infty}\right\|_{L^{q}}=\left\|\rho_{0}\right\|_{L^{q}}$, since $L^{q}$ $(1<q<+\infty)$ is a uniformly convex Banach space, and we can conclude that $\rho\left(t_{i}^{\prime}\right)$ actually strongly converge to $\rho_{\infty}$ in $L^{q}$ (cf. [46, Lemma 3.1.6]), namely, (1.14) holds true.

The uniform-in-time estimate (3.40) yields that for any sequence $\left\{t_{i}\right\} \nearrow+\infty$, there is a subsequence $\left\{t_{i}^{\prime}\right\} \nearrow+\infty$ such that $d\left(t_{i}^{\prime}\right)$ strongly converge to a certain $d_{\infty}$ in $\mathbf{H}^{2}$. Then by (4.1), we see that

$$
\begin{aligned}
0 & \leq\left\|-\Delta d_{\infty}+f\left(d_{\infty}\right)\right\| \\
& \leq\left\|-\Delta d\left(t_{i}^{\prime}\right)+f\left(d\left(t_{i}^{\prime}\right)\right)\right\|+\left\|\Delta d\left(t_{i}^{\prime}\right)-\Delta d_{\infty}\right\|+\left\|f\left(d\left(t_{i}^{\prime}\right)\right)-f\left(d_{\infty}\right)\right\| \\
& \leq\left\|-\Delta d\left(t_{i}^{\prime}\right)+f\left(d\left(t_{i}^{\prime}\right)\right)\right\|+C\left\|d\left(t_{i}^{\prime}\right)-d_{\infty}\right\|_{\mathbf{H}^{2}} \\
& \rightarrow 0, \quad \text { as } t_{i}^{\prime} \nearrow+\infty .
\end{aligned}
$$

Thus, $d_{\infty}$ satisfies the stationary problem (1.16). The $\mathbf{H}^{3}$ convergence follows from (4.2) and the fact that

$$
\begin{aligned}
\left\|\nabla \Delta\left(d\left(t_{i}^{\prime}\right)-d_{\infty}\right)\right\| & \leq\left\|\nabla\left(-\Delta d\left(t_{i}^{\prime}\right)+f\left(d\left(t_{i}^{\prime}\right)\right)\right)\right\|+\left\|\nabla\left(f\left(d\left(t_{i}^{\prime}\right)\right)-f\left(d_{\infty}\right)\right)\right\| \\
& \leq\left\|\nabla\left(-\Delta d\left(t_{i}^{\prime}\right)+f\left(d\left(t_{i}^{\prime}\right)\right)\right)\right\|+C\left\|d\left(t_{i}^{\prime}\right)-d_{\infty}\right\|_{\mathbf{H}^{2}}
\end{aligned}
$$




$$
\rightarrow 0, \quad \text { as } t_{i}^{\prime} \nearrow+\infty
$$

The proof of Theorem 1.2 is complete.

REMARK 4.1.

(1) When the external force $\mathbf{g}$ is a gradient field independent of time, our results show that the velocity field and its time derivative will converge to zero as time goes to infinity, this coincides with the result for the $2 D$ density-dependent incompressible Navier-Stokes equations [45].

(2) For the density function $\rho$, we are only able to obtain the partial result that it will sequentially converge to a certain function in $L^{q}$ norm (differential sequences may have different limit points). One possible sufficient condition for the convergence in time is that $\left\|\rho_{t}\right\|_{H^{-1}} \in L^{1}(0,+\infty)$. On the other hand, we observe that, for any function $\varphi \in H_{0}^{1}(\Omega)$,

$$
\int_{\Omega} \rho_{t} \varphi d x=-\int_{\Omega} \nabla \cdot(\rho v) \varphi d x=\int_{\Omega} \rho v \cdot \nabla \varphi d x \leq \bar{\rho}\|v\|\|\nabla \varphi\|
$$

which yields $\left\|\rho_{t}\right\|_{H^{-1}} \leq C\|v\|$. Hence, if we can prove $\|v\| \in L^{1}(0,+\infty)$, then we get the convergence of $\rho$ as time tends to infinity in the space $H^{-1}$, and thus in $L^{q}$ due to the uniqueness of limit. However, we do not know this $L^{1}$-integrability condition on $v$ from the above proof.

(3) For the director vector $d$, we are able to show the decay of its time derivative and sequential convergence of itself to a steady state that is a solution to the stationary problem (1.16). If we know that problem (1.16) admits a unique solution, then $d$ will converge to it as time goes to infinity. However, in general we cannot expect the uniqueness of solutions to the stationary Ginzburg-Landau equation (1.16) unless, for instance, the parameter $\eta$ in $f(d)$ is sufficiently large.

4.2. Proof of Theorem 1.3. In this subsection, we provide the proof for Theorem 1.3. Now the external force $\mathbf{g}$ is asymptotically autonomous and satisfies (F2) and (1.17). Unlike the previous case with a time-independent force, we shall see that one can obtain convergence for the density function and director vector. The proof is based on an appropriate generalization of the Lojasiewicz-Simon approach for gradient-like systems (cf. e.g., [16]).

Denote

$$
E(d)=\frac{1}{2}\|\nabla d\|^{2}+\int_{\Omega} F(d) d x .
$$

It is straightforward to check that any solution to the stationary problem (1.16) is a critical point of the energy functional $E(d)$, and conversely, that the critical point of $E(d)$ is a solution to (1.16) (cf. [42]). Besides, regularity of solutions to (1.16) has been shown in [25] such that $d$ is smooth on $\Omega$ provided that $d_{0}$ is smooth on $\Gamma$. Below we shall make use the following Eojasiewicz-Simon type inequality (cf. [42]):

Lemma 4.3. Let $\psi$ be a critical point of $E(d)$. There exist constants $\theta \in\left(0, \frac{1}{2}\right)$ and $\beta>0$ depending on $\psi$ such that for any $d \in \mathbf{H}^{2}(\Omega)$ satisfying $\left.d\right|_{\Gamma}=d_{0}(x)$ and $\|d-\psi\|_{\mathbf{H}^{2}}<\beta$, it holds that

$$
\|-\Delta d+f(d)\| \geq|E(d)-E(\psi)|^{1-\theta} .
$$


The uniform estimate (3.40) yields that the set of all limit points of the trajectory $\{d(t): t \geq 0\}$ denoted by

$$
\omega(d):=\bigcap_{s \geq 0} \overline{\left\{d(t) \in \mathbf{H}^{2}(\Omega): t \geq s\right\}}
$$

is non-empty and compact in $\mathbf{H}^{2}(\Omega)$.

Besides, similar to Section 4.1, we can see that each element in $\omega(d)$ is a solution to problem (1.16) and thus is a critical point of $E(d)$. We infer from (3.3) that

$$
\mathcal{E}\left(t_{1}\right)-\mathcal{E}\left(t_{2}\right) \leq C \int_{t_{2}}^{t_{1}}\|\mathbf{g}\|^{2} d t, \quad \forall t_{1}>t_{2}>0 .
$$

Since $\|\mathbf{g}\| \in L^{2}(0,+\infty)$, it follows that $\mathcal{E}(t)$ converges to a certain constant $\mathcal{E}_{\infty}$ as $t \rightarrow+\infty$.

Recalling the convergence of the velocity field (4.1), we deduce that $E(d)$ is indeed a constant on $\omega(d)$ such that $E(\psi)=\lambda^{-1} \mathcal{E}_{\infty}$, for all $\psi \in \omega(d)$. Due to Lemma 4.3, for every $\psi \in \omega(d)$, there exist some $\beta_{\psi}$ and $\theta_{\psi} \in\left(0, \frac{1}{2}\right)$ that may depend on $\psi$ such that the inequality (4.9) holds for

$$
d \in \mathbf{B}_{\beta_{\psi}}(\psi):=\left\{d \in \mathbf{H}^{2}(\Omega):\left.d\right|_{\Gamma}=d_{0},\|d-\psi\|_{\mathbf{H}^{2}}<\beta_{\psi}\right\}
$$

and $|E(d)-E(\psi)| \leq 1$. The union of balls $\left\{\mathcal{B}_{\beta_{\psi}}(\psi): \psi \in \omega(d)\right\}$ forms an open cover of $\omega(d)$ and, due to the compactness of $\omega(d)$, we can find a finite sub-cover $\left\{\mathbf{B}_{\beta_{i}}\left(\psi_{i}\right)\right.$ : $i=1,2, \ldots, m\}$ where the constants $\beta, \theta$ corresponding to $\psi_{i}$ in Lemma 4.3 are indexed by $i$. From the definition of $\omega(d)$, we know that there exist a sufficient large $t_{0}$ such that

$$
d(t) \in \mathcal{U}:=\cup_{i=1}^{m} \mathcal{B}_{\beta_{i}}\left(\psi_{i}\right), \quad \text { for } t \geq t_{0} .
$$

Taking $\theta=\min _{i=1}^{m}\left\{\theta_{i}\right\} \in\left(0, \frac{1}{2}\right)$, we get for all $t \geq t_{0}$,

$$
\|-\Delta d(t)+f(d(t))\| \geq\left|E(d(t))-\lambda^{-1} \mathcal{E}_{\infty}\right|^{1-\theta} .
$$

Denote

$$
\begin{aligned}
\mathcal{Y}(t)^{2} & =\frac{\nu}{2}\|\nabla v(t)\|^{2}+\lambda \gamma\|\Delta d(t)-f(d(t))\|^{2}, \\
z(t) & =\int_{t}^{+\infty}\|\mathbf{g}(\tau)\|^{2} d \tau .
\end{aligned}
$$

Assumption (1.17) implies that

$$
z(t) \leq C(1+t)^{-(1+\xi)}, \quad \text { for } t \geq 0 .
$$

Then by the basic energy inequality (3.3), we get

$$
\begin{aligned}
\mathcal{E}(t)-\mathcal{E}_{\infty} & \geq \int_{t}^{+\infty} \mathcal{Y}(\tau)^{2} d \tau-\frac{C_{P}^{2} \bar{\rho}^{2}}{2 \nu} z(t) \\
& \geq \int_{t}^{+\infty} \mathcal{Y}(\tau)^{2} d \tau-C(1+t)^{-(1+\xi)} .
\end{aligned}
$$


On the other hand, using (4.11), the uniform estimates (3.27), and the fact $\frac{1}{1-\theta}<2$, we obtain that

$$
\begin{aligned}
\left|\mathcal{E}(t)-\mathcal{E}_{\infty}\right| & \leq \frac{1}{2}\|v\|^{2}+\lambda\left|E(d)-\lambda^{-1} \mathcal{E}_{\infty}\right| \\
& \leq C\|\nabla v\|^{\frac{1}{1-\theta}}+\lambda\|-\Delta d(t)+f(d(t))\|^{\frac{1}{1-\theta}} \\
& \leq C \mathcal{Y}(t)^{\frac{1}{1-\theta}}, \quad \forall t \geq t_{0} .
\end{aligned}
$$

Take

$$
\zeta=\min \left\{\theta, \frac{\xi}{2(1+\xi)}\right\} \in\left(0, \frac{1}{2}\right)
$$

It is easy to check that

$$
\begin{aligned}
\int_{t}^{+\infty}(1+\tau)^{-2(1+\xi)(1-\zeta)} d \tau & \leq \int_{t}^{+\infty}(1+\tau)^{-(2+\xi)} d \tau \\
& \leq(1+t)^{-(1+\xi)}, \quad \forall t \geq 0
\end{aligned}
$$

We now set

$$
Z(t)=\mathcal{Y}(t)+(1+t)^{-(1+\xi)(1-\zeta)} .
$$

Since $\lim _{t \rightarrow+\infty} \mathcal{Y}(t)=0$ (see (4.1)), it follows from (4.12), (4.13), and (4.14) that

$$
\begin{aligned}
\int_{t}^{+\infty} Z(\tau)^{2} d \tau & \leq C \mathcal{Y}(t)^{\frac{1}{1-\theta}}+C(1+t)^{-(1+\xi)} \\
& \leq C \mathcal{Y}(t)^{\frac{1}{1-\zeta}}+C(1+t)^{-(1+\xi)} \\
& \leq C Z(t)^{\frac{1}{1-\zeta}}, \quad \forall t \geq t_{0}
\end{aligned}
$$

Recall the following result (cf. [16, Lemma 4.1] or [11, Lemma 7.1]).

Lemma 4.4. Let $\zeta \in\left(0, \frac{1}{2}\right)$. Assume that $Z \geq 0$ be a measurable function on $(0,+\infty)$, $Z \in L^{2}\left(\mathbb{R}^{+}\right)$, and there exist $C>0$ and $t_{0} \geq 0$ such that

$$
\int_{t}^{+\infty} Z(\tau)^{2} d \tau \leq C Z(t)^{\frac{1}{1-\zeta}}, \quad \text { for a.e. } t \geq t_{0}
$$

Then $Z \in L^{1}\left(t_{0},+\infty\right)$.

We conclude from (4.15) and Lemma 4.4 that

$$
\int_{t_{0}}^{+\infty} Z(t)<+\infty
$$

Since $\xi>0$, it holds that

$$
\begin{aligned}
\int_{t_{0}}^{+\infty}(1+t)^{-(1+\xi)(1-\zeta)} d t & \leq \int_{t_{0}}^{+\infty}(1+t)^{-\frac{1}{2}(2+\xi)} d t \\
& \leq 2\left(1+t_{0}\right)^{-(1+\xi)}<+\infty, \text { for } t_{0}>0
\end{aligned}
$$


which implies that

$$
\int_{t_{0}}^{+\infty}\|\nabla v(t)\|+\|\Delta d(t)-f(d(t))\| d t<+\infty .
$$

On the other hand, it follows from equation (1.3) that

$$
\begin{aligned}
\left\|d_{t}\right\| & \leq C\left(\|v\|_{\mathbf{L}^{4}}\|\nabla d\|_{\mathbf{L}^{4}}+\|-\Delta d+f(d)\|\right) \\
& \leq C(\|\nabla v\|+\|-\Delta d+f(d)\|) .
\end{aligned}
$$

As a consequence,

$$
\int_{t_{0}}^{\infty}\left\|d_{t}(t)\right\| d t<+\infty
$$

which easily implies that as $t \rightarrow+\infty, d(t)$ converges strongly in $\mathbf{L}^{2}(\Omega)$. By compactness of $d(t)$ in $\mathbf{H}^{2}(\Omega)$, we deduce that

$$
\lim _{t \rightarrow+\infty}\left\|d(t)-d_{\infty}\right\|_{\mathbf{H}^{2}}=0
$$

where $d_{\infty}$ is a solution to problem (1.16). Recalling the uniform estimate (3.40), it holds that

$$
\begin{aligned}
\left\|\nabla \Delta d-\nabla \Delta d_{\infty}\right\| & \leq\left\|\nabla\left(\Delta d-\Delta d_{\infty}-f(d)+f\left(d_{\infty}\right)\right)\right\|+\left\|\nabla\left(f(d)-f\left(d_{\infty}\right)\right)\right\| \\
& \leq\|\nabla(\Delta d-f(d))\|+C\left\|d-d_{\infty}\right\|_{\mathbf{H}^{2}} .
\end{aligned}
$$

The above estimate together with (4.2) and (4.19) yields

$$
\lim _{t \rightarrow+\infty}\left\|d(t)-d_{\infty}\right\|_{\mathbf{H}^{3}}=0 .
$$

It follows from Remark 4.1 and (4.16) that $\left\|\rho_{t}\right\|_{H^{-1}} \in L^{1}\left(t_{0},+\infty\right)$. Thus $\rho(t)$ converges strongly in $H^{-1}$ as $t \rightarrow+\infty$. By an argument similar to that in the proof of Theorem 1.2 , we conclude that

$$
\lim _{t \rightarrow+\infty}\left\|\rho(t)-\rho_{\infty}\right\|_{L^{q}}=0, \quad q \in(1,+\infty)
$$

Next, we prove the convergence rate. Denote

$$
\mathcal{K}(t)=\mathcal{E}(t)-\mathcal{E}_{\infty}+\frac{C_{P}^{2} \bar{\rho}^{2}}{2 \nu} \int_{t}^{+\infty}\|\mathbf{g}(\tau)\|^{2} d \tau
$$

It follows from the basic energy inequality (3.3) that

$$
\frac{d}{d t} \mathcal{K}(t)+\mathcal{Y}(t)^{2} \leq 0
$$

Thus, $\mathcal{K}(t)$ is decreasing on $[0,+\infty)$ and $\mathcal{K}(t) \rightarrow 0$ as $t \rightarrow+\infty$. Recalling the definition of $t_{0}$, for $t \geq t_{0}$, we deduce from (1.17) and (4.13) that

$$
\begin{aligned}
\mathcal{K}(t)^{2(1-\zeta)} & \leq C \mathcal{Y}(t)^{\frac{2(1-\zeta)}{1-\theta}}+C(1+t)^{-2(1-\zeta)(1+\xi)} \\
& \leq C \mathcal{Y}(t)^{2}+C(1+t)^{-2(1-\zeta)(1+\xi)}
\end{aligned}
$$




$$
\leq-C \frac{d}{d t} \mathcal{K}(t)+C(1+t)^{-2(1-\zeta)(1+\xi)},
$$

where we have used the fact that

$$
\frac{2(1-\zeta)}{1-\theta} \geq 2
$$

It follows from the ordinary differential inequality (4.23) and [2, Lemma 2.6] that

$$
\mathcal{K}(t) \leq C(1+t)^{-\iota}, \quad \forall t \geq t_{0}
$$

with the exponent given by

$$
\iota=\min \left\{\frac{1}{1-2 \zeta}, 1+\xi\right\}=\min \left\{\frac{1}{1-2 \theta}, 1+\xi\right\} .
$$

We infer from (4.22) that for any $t \geq t_{0}$,

$$
\int_{t}^{2 t} \mathcal{Y}(\tau) d \tau \leq t^{\frac{1}{2}}\left(\int_{t}^{2 t} \mathcal{Y}(\tau)^{2} d \tau\right)^{\frac{1}{2}} \leq C t^{\frac{1}{2}} \mathcal{K}(t)^{\frac{1}{2}} \leq C(1+t)^{\frac{1-\iota}{2}},
$$

where

$$
\kappa=\frac{\iota-1}{2}=\min \left\{\frac{\theta}{1-2 \theta}, \frac{\xi}{2}\right\}>0 .
$$

It holds that

$$
\int_{t}^{+\infty} \mathcal{Y}(\tau) d \tau \leq \sum_{j=0}^{+\infty} \int_{2^{j} t}^{2^{j+1} t} \mathcal{Y}(\tau) d \tau \leq C \sum_{j=0}^{+\infty}\left(2^{j} t\right)^{-\kappa} \leq C(1+t)^{-\kappa}, \quad \forall t \geq t_{0} .
$$

Then by (4.17), we get

$$
\int_{t}^{+\infty}\left\|d_{t}(\tau)\right\| d \tau \leq \int_{t}^{+\infty} \mathcal{Y}(\tau) d \tau \leq C(1+t)^{-\kappa}, \quad \forall t \geq t_{0},
$$

which together with (3.27) yields the convergence rate of $d$ in $\mathbf{L}^{2}$ :

$$
\left\|d(t)-d_{\infty}\right\| \leq C(1+t)^{-\kappa}, \quad \forall t \geq 0 .
$$

Besides, using the Poincaré inequality, we infer from Remark 4.1 and (4.25) that

$$
\begin{aligned}
\int_{t}^{+\infty}\left\|\rho_{t}(\tau)\right\|_{H^{-1}} & \leq C \int_{t}^{+\infty}\|v(\tau)\| d \tau \leq C \int_{t}^{+\infty}\|\mathcal{Y}(\tau)\| d \tau \\
& \leq C(1+t)^{-\kappa}, \quad \forall t \geq 0
\end{aligned}
$$

which implies

$$
\left\|\rho(t)-\rho_{\infty}\right\|_{H^{-1}} \leq C(1+t)^{-\kappa}, \quad \forall t \geq 0 .
$$

Taking advantage of the lower-order convergence rate of the director $d$ (4.26), we are able to obtain decay estimate for $v$ as well as a higher-order convergence rate 
on $d$. For this purpose, we make use the fact $-\Delta d_{\infty}+f\left(d_{\infty}\right)=0$ and test (1.4) by $\lambda\left(-\Delta\left(d-d_{\infty}\right)+f(d)-f\left(d_{\infty}\right)+d-d_{\infty}\right)$ to get

$$
\begin{aligned}
& \frac{d}{d t}(\left.\frac{\lambda}{2}\left\|\nabla\left(d-d_{\infty}\right)\right\|^{2}+\frac{\lambda}{2}\left\|d-d_{\infty}\right\|^{2}+\lambda \int_{\Omega} F(d)-F\left(d_{\infty}\right)-f\left(d_{\infty}\right) \cdot\left(d-d_{\infty}\right) d x\right) \\
&+\lambda \gamma\|-\Delta d+f(d)\|^{2}+\lambda \gamma\left\|\nabla\left(d-d_{\infty}\right)\right\|^{2}+\lambda \int_{\Omega}(v \cdot \nabla d) \cdot \Delta d d x \\
&=-\lambda \int_{\Omega}(v \cdot \nabla d) \cdot\left(d-d_{\infty}\right) d x-\lambda \gamma \int_{\Omega}\left(f(d)-f\left(d_{\infty}\right)\right) \cdot\left(d-d_{\infty}\right) d x
\end{aligned}
$$

Using the uniform estimate (3.40) and the Sobolev embeddings, we can estimate the right-hand side of (4.27) as follows:

$$
\text { R.H.S of }(4.27) \leq \frac{\nu}{4}\|\nabla v\|^{2}+C\left\|d-d_{\infty}\right\|^{2} .
$$

Adding (4.27) with (3.4) and (3.5), we infer from (4.28) that

$$
\frac{d}{d t} \mathcal{Q}(t)+C_{1}\left(A(t)+\left\|\nabla\left(d-d_{\infty}\right)\right\|^{2}\right) \leq C_{2}\left(\left\|d-d_{\infty}\right\|^{2}+\|\mathbf{g}\|^{2}\right)
$$

where

$$
\begin{gathered}
\mathcal{Q}(t)=\frac{1}{2} \int_{\Omega} \rho(t)|v(t)|^{2} d x+\frac{\lambda}{2}\left\|\nabla\left(d(t)-d_{\infty}\right)\right\|^{2}+\frac{\lambda}{2}\left\|d(t)-d_{\infty}\right\|^{2} \\
+\lambda \int_{\Omega} F(d(t))-F\left(d_{\infty}\right)-f\left(d_{\infty}\right) \cdot\left(d(t)-d_{\infty}\right) d x
\end{gathered}
$$

By the uniform estimate (3.40) and Taylor's formula, it is easy to see that

$$
\left|\int_{\Omega} F(d)-F\left(d_{\infty}\right)-f\left(d_{\infty}\right)\left(d-d_{\infty}\right) d x\right| \leq C_{3}\left\|d-d_{\infty}\right\|^{2}
$$

which implies

$$
\mathcal{Q}(t)+C_{3}\left\|d(t)-d_{\infty}\right\|^{2} \geq \frac{1}{2} \int_{\Omega} \rho(t)|v(t)|^{2} d x+\frac{\lambda}{2}\left\|\nabla\left(d(t)-d_{\infty}\right)\right\|^{2} .
$$

Since $A(t)$ is uniformly bounded in time, (3.18) can be rewritten as

$$
\frac{d}{d t} A(t) \leq C_{4} A(t)+C_{5}\|\mathbf{g}\|^{2}
$$

We deduce from (4.29), (4.30), and (4.31) that

$$
\frac{d}{d t} \mathcal{Q}_{1}(t)+C_{6} \mathcal{Q}_{1}(t) \leq C_{7}\left(\left\|d-d_{\infty}\right\|^{2}+\|\mathbf{g}\|^{2}\right),
$$

where $\mathcal{Q}_{1}(t)=\mathcal{Q}(t)+\frac{C_{1}}{2 C_{4}} A(t)$. As a consequence,

$$
\begin{aligned}
\mathcal{Q}_{1}(t) & \leq e^{-C_{6} t}\left(\mathcal{Q}_{1}(0)+C_{7} \int_{0}^{t} e^{C_{6} \tau}\left(\left\|d(\tau)-d_{\infty}\right\|^{2}+\|\mathbf{g}(\tau)\|^{2}\right) d \tau\right) \\
& \leq \mathcal{Q}_{1}(0) e^{-C_{6} t}+C e^{-\frac{C_{6} t}{2}} \int_{0}^{\frac{t}{2}}\left(\left\|d(\tau)-d_{\infty}\right\|^{2}+\|\mathbf{g}(\tau)\|^{2}\right) d \tau
\end{aligned}
$$




$$
\begin{aligned}
& +C\left(\sup _{s \in\left[\frac{t}{2}, t\right]}\left\|d(s)-d_{\infty}\right\|^{2}\right) e^{-C_{6} t} \int_{\frac{t}{2}}^{t} e^{C_{6} \tau} d \tau+C \int_{\frac{t}{2}}^{+\infty}\|\mathbf{g}(t)\|^{2} d \tau \\
& \leq \mathcal{Q}_{1}(0) e^{-C_{6} t}+e^{-\frac{C_{6} t}{2}}\left(C \int_{0}^{\frac{t}{2}}(1+\tau)^{-2 \kappa} d \tau+C\right) \\
& \quad+C\left(1+\frac{t}{2}\right)^{-2 \kappa}+C\left(1+\frac{t}{2}\right)^{-(1+\xi)}+\quad \forall t \geq 0 .
\end{aligned}
$$

Recalling the definitions of $A(t), \mathcal{Q}_{1}(t), \mathcal{Q}(t)$, we conclude from (4.33), (4.30), (4.26), and (3.12) that

$$
\|v(t)\|_{\mathbf{H}^{1}}+\left\|d(t)-d_{\infty}\right\|_{\mathbf{H}^{1}}+\|\Delta d(t)-f(d(t))\| \leq C(1+t)^{-\kappa}, \quad \forall t \geq 0 .
$$

By the elliptic estimate

$$
\begin{aligned}
\left\|d-d_{\infty}\right\|_{\mathbf{H}^{2}} & \leq C\left\|\Delta\left(d-d_{\infty}\right)\right\| \leq C\|\Delta d-f(d)\|+C\left\|f(d)-f\left(d_{\infty}\right)\right\| \\
& \leq C\|\Delta d-f(d)\|+\left\|d-d_{\infty}\right\|_{\mathbf{H}^{1}}
\end{aligned}
$$

we get

$$
\left\|d(t)-d_{\infty}\right\|_{\mathbf{H}^{2}} \leq C(1+t)^{-\kappa}, \quad \forall t \geq 0 .
$$

The proof of Theorem 1.3 is complete.

REMARK 4.2. If the velocity field $v$ decays fast enough, we can obtain uniformin-time $W^{1, r}$-estimate for $\rho(1<r<+\infty)$. For any $q \in(1,+\infty)$, we infer from (3.48),

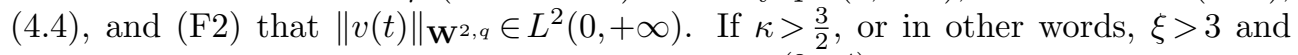
$\theta \in\left(\frac{3}{8}, \frac{1}{2}\right)$, we just take $q \in\left(\frac{4 \kappa-4}{2 \kappa-3},+\infty\right)$ such that $\frac{(2 q-4) \kappa}{3 q-4}>1$. It then follows from (4.34) that

$$
\begin{aligned}
& \int_{0}^{+\infty}\|\nabla v(t)\|_{\mathbf{L}^{\infty}} d t \\
\leq & C \int_{0}^{+\infty}\|v(t)\|_{\mathbf{W}^{2, q}}^{\frac{q}{2(q-1)}}\|\nabla v(t)\|^{\frac{q-2}{2(q-1)}} d t \\
\leq & C\left(\int_{0}^{+\infty}\|\nabla v(t)\|^{\frac{2 q-4}{3 q-4}} d t\right)^{\frac{3 q-4}{4(q-1)}}\left(\int_{0}^{+\infty}\|v(t)\|_{\mathbf{W}^{2, q}}^{2} d t\right)^{\frac{q}{4(q-1)}} \\
\leq & C\left(\int_{0}^{+\infty}(1+t)^{-\frac{(2 q-4) \kappa}{3 q-4}} d t\right)^{\frac{3 q-4}{4(q-1)}} \\
\leq & C .
\end{aligned}
$$

Therefore, by (2.1), we obtain that $\|\rho(t)\|_{W^{1, r}} \leq C\left\|\rho_{0}\right\|_{W^{1, r}}$ for $t \geq 0$.

Acknowledgment. The authors would like to thank the referee for calling their attention to related works in the literature on this topic. X. Hu's research was partially supported by the National Science Foundation. H. Wu was partially supported by National Science Foundation of China grant 11001058, SRFDP, and "Chen Guang" project supported by the Shanghai Municipal Education Commission and the Shanghai Education Development Foundation. 


\section{REFERENCES}

[1] S.N. Antontsev, A.V. Kazhikhov, and V.N. Monakhov, Boundary Value Problems in Mechanics of Non-Homogeneous Fluids, Stud. Math. Appl., North-Holland, 22, 1990.

[2] I. Ben Hassen, Decay estimates to equilibrium for some asymptotically autonomous semilinear evolution equations, Asymptot. Anal., 69(1-2), 31-44, 2010.

[3] S. Bosia, Well-posedness and long term behavior of a simplified Ericksen-Leslie nonautonomous system for nematic liquid crystal flow, Commun. Pure Appl. Anal., 11, 407$441,2012$.

[4] Y.M. Chu, X.G. Liu, and X. Liu, Strong solutions to the compressible liquid crystal system, Pacific J. Math., 257(1), 37-52, 2012.

[5] Y.M. Chu, W.Y. Ma, and X.G. Liu, Long-time behavior of solutions to the compressible liquid crystals, (in Chinese), Sci. Sin. Math., 42(2), 107-118, 2012.

[6] B. Climent-Ezquerra, F. Guillen-González, and M. Moreno-Iraberte, Regularity and time periodicity for a nematic liquid crystal model, Nonlin. Anal., 71, 530-549, 2009.

[7] M. Dai, J. Qing, and M. Schonbek, Regularity of solutions to the liquid crystals systems in $\mathbb{R}^{2}$ and $\mathbb{R}^{3}$, Nonlin., 25, 513-532, 2012.

[8] R. Danchin, Density-dependent incompressible fluids in bounded domains, J. Math. Fluid Mech., 8, 333-381, 2006.

[9] P.G. de Gennes, The Physics of Liquid Crystals, Oxford, 1974.

[10] J. Ericksen, Conservation laws for liquid crystals, Trans. Soc. Rheol., 5, 22-34, 1961.

[11] E. Feireisl and F. Simondon, Convergence for semilinear degenerate parabolic equations in several space dimensions, J. Dyn. Diff. Equ., 12(3), 647-673, 2000.

[12] M. Grasselli and $\mathrm{H}$. Wu, Long-time behavior for a hydrodynamic model on nematic liquid crystal flows with asymptotic stabilizing boundary condition and external force, preprint, 2011, arXiv:1111.1285.

[13] M.A. Jendoubi, A simple unified approach to some convergence theorem of L. Simon, J. Func. Anal., 153, 187-202, 1998.

[14] F. Jiang and Z. Tan, Global weak solution to the flow of liquid crystals system, Math. Meth. Appl. Sci., 32, 2243-2266, 2009.

[15] X. Hu and D. Wang, Global solution to the three-dimensional incompressible flow of liquid crystals, Commun. Math. Phys., 296, 861-880, 2010.

[16] S.Z. Huang and P. Takáč, Convergence in gradient-like systems which are asymptotically autonomous and analytic, Nonlin. Anal., 46, 675-698, 2001.

[17] T. Huang and C.Y. Wang, Blow up criterion for nematic liquid crystal flows, Commun. Part. Diff. Equ., 37(5), 875-884, 2012.

[18] O.A. Ladyzhenskaya and V.A. Solonnikov, Unique solvability of an initial and boundary value problem for viscous incompressible non-homogeneous fluids, J. Soviet. Math., 9, 697-749, 1978.

[19] F. Leslie, Some constitutive equations for liquid crystals, Arch. Rat. Mech. Anal., 28, 265-283, 1968.

[20] F.M. Leslie, Theory of flow phenomena in liquid crystals, in Advances in Liquid Crystals, G. Brown ed., Academic Press, New York, 4, 1-81, 1979.

[21] X.L. Li and D.H. Wang, Global strong solution to the density-dependent incompressible flow of liquid crystals, Trans. AMS, to appear, 2012.

[22] F.H. Lin, Nonlinear theory of defects in nematic liquid crystals: Phase transitions and flow phenomena, Commun. Pure Appl. Math., XLII, 789-814, 1989.

[23] F.H. Lin and Q. Du, Ginzburg-Landau vortices: Dynamics, pinning, and hysteresis, SIAM J. Math. Anal., 28, 1265-1293, 1997.

[24] F.H. Lin, J.Y. Lin, and C.Y. Wang, Liquid crystal flows in two dimensions, Arch. Rat. Mech. Anal., 197(1), 297-336, 2010.

[25] F.H. Lin and C. Liu, Nonparabolic dissipative system modeling the flow of liquid crystals, Commun. Pure Appl. Math., XLVIII, 501-537, 1995.

[26] F.H. Lin and C. Liu, Partial regularities of the nonlinear dissipative systems modeling the flow of liquid crystals, Disc. Cont. Dyn. Sys., 2, 1-23, 1996.

[27] F.H. Lin and C.Y. Wang, On the uniqueness of heat flow of harmonic maps and hydrodynamic flow of nematic liquid crystals, Chin. Ann. Math. Ser. B, 31(6), 921-938, 2010.

[28] C. Liu and N.J. Walkington, Approximation of liquid crystal flows, SIAM J. Num. Anal., 37, 725-741, 2000.

[29] C. Liu and J. Shen, On liquid crystal flows with free-slip boundary conditions, Disc. Cont. Dyn. Sys., 7, 307-318, 2001.

[30] C. Liu and N.J. Walkington, Mixed methods for the approximation of liquid crystal flows, Math. 
Model. Num. Anal., 36(2), 205-222, 2002.

[31] L.M. Liu and X.G. Liu, A blow-up criterion of strong solutions to the compressible liquid crystals system, Chinese J. Contemp. Math., 32, 211-224, 2011.

[32] X.G. Liu and J. Qing, Globally weak solutions to the flow of compressible liquid crystals system, Disc. Cont. Dyn. Sys., 33(2), 757-788, 2013.

[33] X.G. Liu and Z. Zhang, Existence of the flow of liquid crystals system (in Chinese), Chinese Ann. Math. A., 30(1), 1-20, 2009.

[34] X.G. Liu, L.M. Liu, and Y.H. Hao, Existence of strong solutions for the compressible EricksenLeslie model, arXiv:1106.6140, preprint, 2011.

[35] H. Petzeltová, E. Rocca, and G. Schimperna, On the long-time behavior of some mathematical models for nematic liquid crystals, Calc. Var. Part. Diff. Equ., 2012. online first, DOI: 10.1007/s00526-012-0496-1.

[36] C. Simader and H. Sohr, A new approach to the Helmholtz decomposition and the Neumann problem in $L^{q}$ spaces for bounded and exterior domains, Mathematical Problems Relating to the Navier-Stokes Equation, in: Ser. Adv. Math. Appl. Sci., World Sci. Publ., River Edge, NJ, 11, 1-35, 1992.

[37] L. Simon, Asymptotics for a class of nonlinear evolution equation with applications to geometric problems, Ann. of Math., 118, 525-571, 1983.

[38] R. Temam, Navier-Stokes Equations and Nonlinear Functional Analysis, SIAM, 1983.

[39] C.Y. Wang, Well-posedness for the heat flow of harmonic maps and the liquid crystal flow with rough initial data, Arch. Rat. Mech. Anal., 200(1), 1-19, 2011.

[40] D.H. Wang and C. Yu, Global weak solution and large-time behavior for the compressible flow of liquid crystals, Arch. Rat. Mech. Anal., 204(3), 881-915, 2012.

[41] H.Y. Wen and S.J. Ding, Solutions of incompressible hydrodynamic flow of liquid crystals, Nonlin. Anal. Real World Appl., 12(3), 1510-1531, 2011.

[42] $\mathrm{H}$. Wu, Long-time behavior for nonlinear hydrodynamic system modeling the nematic liquid crystal flows, Disc. Cont. Dyn. Sys., 26(1), 379-396, 2010.

[43] J.K. Xu and Z. Tan, Global existence of the finite energy weak solutions to a nematic liquid crystals model, Math. Meth. Appl. Sci., 34(8), 929-938, 2011.

[44] X. Xu and Z. Zhang, Global regularity and uniqueness of weak solution for the 2-D liquid crystal flows, J. Diff. Equ., 252, 1169-1181, 2012.

[45] K. Zhao, Large time behavior for density dependent incompressible Navier-Stokes equations on bounded domains, J. Math. Fluid Mech., 14(3), 471-483, 2012.

[46] S. Zheng, Nonlinear Evolution Equations, Pitman series Monographs and Survey in Pure and Applied Mathematics, Chapman \& Hall/CRC, Boca Raton, Florida, 133, 2004. 